\title{
Imaging gravity waves in lower stratospheric AMSU-A radiances, Part 1: Simple forward model
}

\author{
S. D. Eckermann ${ }^{1}$ and D. L. $\mathbf{W u}^{2}$ \\ ${ }^{1}$ E. O. Hulburt Center for Space Research, Naval Research Laboratory, Washington, D.C., USA \\ ${ }^{2}$ Jet Propulsion Laboratory, California Institute of Technology, Pasadena, California, USA
}

Received: 30 November 2005 - Published in Atmos. Chem. Phys. Discuss.: 27 March 2006

Revised: 1 August 2006 - Accepted: 9 August 2006 - Published: 14 August 2006

\begin{abstract}
Using a simplified model of in-orbit radiance acquisition by the Advanced Microwave Sounding Unit (AMSU-A), we derive three-dimensional temperature weighting functions for Channel 9 measurements (peaking at $\sim 60-90 \mathrm{hPa})$ at all 30 cross-track beam positions and use them to investigate the sensitivity of these radiances to gravity waves. The vertical widths of the weighting functions limit detection to waves with vertical wavelengths of $\gtrsim 10 \mathrm{~km}$, with slightly better vertical wavelength sensitivity at the outermost scan angles due to the limb effect. Fourier Transforms of two-dimensional cross-track weighting functions reveal optimal sensitivity to cross-track wavelengths at the near-nadir scan angles, where horizontal measurement footprints are smallest. This sensitivity is greater for the AMSU-A on the Aqua satellite than for the identical instruments on the NOAA meteorological satellites, due to a lower orbit altitude and thus smaller horizontal footprints from antenna spreading. Small cross-track asymmetries in the radiance response to gravity waves are found that peak at the mid-range scan angles, with more symmetric responses at near-nadir and far off-nadir scan angles. Three-dimensional simulations show gravity wave oscillations imaged in horizontal AMSU-A radiance maps swept out by the scan pattern and satellite motion. A distorting curvature is added to imaged wave phase lines due to vertical variations in weighting function peaks with cross-track scan angle. This wave distortion is analogous to the well-known "limb darkening" and "limb brightening" of microwave radiances acquired from purely vertical background temperature profiles by crosstrack scanners. Waves propagating along track are more visible in these images at the outermost scan angles than those propagating cross track, due to oversampling and narrower widths of the horizontal measurement footprints in the along track direction. Based on nominal noise floors and repre-
\end{abstract}

Correspondence to: S. D. Eckermann

(stephen.eckermann@nrl.navy.mil) sentative lower stratospheric wave temperature amplitudes, our modeling indicates that Channel 9 AMSU-A radiances can resolve and horizontally image gravity waves with horizontal wavelengths of $\gtrsim 150 \mathrm{~km}$ and vertical wavelengths of $\gtrsim 10 \mathrm{~km}$.

\section{Introduction}

While satellite remote sensors have revolutionized our understanding of the global-scale dynamics and chemistry of the stratosphere, until recently these instruments have lacked the necessary horizontal and vertical resolutions to resolve gravity waves. Thus, the observational record on stratospheric gravity wave dynamics to date has relied mostly on suborbital observations at scattered locations around the globe (e.g., Eckermann et al., 1995; Wang and Geller, 2003). As a result, our understanding of gravity waves on a global-scale is considerably poorer than of the larger-scale stratospheric dynamics (Fritts and Alexander, 2003). Since gravity wave dynamics drive important aspects of the global stratospheric circulation, climate and chemical state (e.g., Alexander and Rosenlof, 2003; Mann et al., 2005), this lack of data represents an important gap in our knowledge.

During the mid-to-late 1990s, advances in limb sounding technology at infrared, microwave and radio wavelengths yielded high-resolution satellite radiances that, for the first time, explicitly resolved some longer wavelength gravity wave oscillations in the stratosphere (e.g., Fetzer and Gille, 1994; Wu and Waters, 1996b; Eckermann and Preusse, 1999; Tsuda et al., 2000). While these data have provided valuable glimpses into the global morphology of stratospheric gravity waves, they have also proven challenging to analyze. The narrow observational wavelength windows within which gravity waves are visible to these instruments vary with channel, orbit position and viewing direction, while the waves themselves vary in wavelength as they propagate through and

Published by Copernicus GmbH on behalf of the European Geosciences Union. 
are refracted by the background atmosphere. Thus different waves are constantly moving into and out of the instrument's "visibility window," complicating interpretation of measured wave variances, particularly their variations with time, altitude and geographical location (Alexander, 1998; Preusse et al., 2000; Jiang et al., 2004; Wu et al., 2006).

Furthermore, the satellite gravity wave data acquired to date often resemble a denser global distribution of suborbital measurements, in the sense of providing only onedimensional cross sections through three-dimensional wave fields. For example, some limb sensors return highresolution vertical temperature profiles with wave oscillations superimposed, but separated too far apart horizontally to resolve horizontal structure (Eckermann and Preusse, 1999; Tsuda et al., 2000). Thus these data provide only vertical profiles of wave oscillations, similar to radiosonde and rocketsonde data. Similarly, limb-tracking measurements from the Microwave Limb Sounder (MLS) on the Upper Atmosphere Research Satellite (UARS) return high-resolution radiances from a given altitude that resolve wave fluctuations along the orbital track (Wu and Waters, 1996b; Jiang et al., 2004), similar to in situ aircraft data. Many fundamental gravity wave properties, such as vertical fluxes of horizontal pseudomomentum densities, cannot be quantified from one-dimensional profiles alone. Thus, we require new satellite observations that can resolve gravity wave oscillations in two or three spatial dimensions. Here we investigate whether two-dimensional maps of lower stratospheric radiances acquired by the Advanced Microwave Sounding UnitA (AMSU-A) can horizontally image gravity waves.

AMSU-A, described in Sect. 2, is the latest in a series of cross-track scanning satellite microwave radiometers. It consolidates over 30 years of accumulated experience and technological development in passive microwave remote sensing of atmospheric temperatures from space, beginning with the Nimbus E Microwave Sounder (NEMS) on the Nimbus 5 satellite. NEMS included three narrowband temperature channels within the $50-60 \mathrm{GHz} \mathrm{O}_{2}$ thermal band whose vertical weighting functions peaked at $\sim 4,10$ and $17 \mathrm{~km}$ altitude (Waters et al., 1975). While NEMS viewed only in the nadir, it was realized that the receiving antenna could be scanned across the satellite track to acquire radiances at a series of other atmospheric locations either side of the subsatellite point. The first instrument with this scanning capability was the Scanning Microwave Spectrometer (SCAMS) on Nimbus 6, which performed sequential "step and stare" measurements at 13 cross-track off-nadir viewing angles distributed symmetrically out to $\pm 1200 \mathrm{~km}$ either side of the subsatellite point (Grody and Pellegrino, 1977). SCAMS was superseded by the Microwave Sounding Unit (MSU), which acquired data in a similar fashion at 11 cross-track beam positions. MSU instruments flew on the NOAA-6 through NOAA-14 meteorological satellites.

SCAMS and MSU used antennas with beamwidths that translated into half-power horizontal measurement footprints at the ground with diameters of $>100 \mathrm{~km}$ at nadir and nearer 200-300 km at the off-nadir angles (Grody and Pellegrino, 1977; Kidder and Vonder Haar, 1995). Since extratropical gravity waves have horizontal wavelengths in the $\sim 5-500 \mathrm{~km}$ range, these instruments could not horizontally image gravity wave perturbations in their acquired radiances, since their footprint "pixels" were too broad. Conversely, the AMSU-A receiving antenna has a narrower antenna polar diagram that yields smaller surface footprints (see Fig. 1 of Kidder et al., 2000). Furthermore, it has many more channels, including 6 stratospheric temperature channels, and improved radiometric accuracy. All these improvements over MSU mean that AMSU-A might resolve and image long wavelength gravity waves oscillations in its stratospheric radiances.

$\mathrm{Wu}$ (2004) investigated this possibility experimentally by extracting and analyzing along-track perturbations in AMSU-A stratospheric radiances at various cross-track scan angles. Maps of along-track Channel 13 radiance variances (peaking at $\sim 5 \mathrm{hPa}$ ) in the Southern Hemisphere showed enhancements over land masses and within strong vortex winds that agreed well with variances in along-track UARS MLS limb radiances acquired at a similar altitude and time of year. This strongly suggests that AMSU-A stratospheric radiances do resolve some gravity wave oscillations.

$\mathrm{Wu}$ and Zhang (2004) used this analysis technique to isolate AMSU-A stratospheric radiance perturbations during 19-21 January 2003, when strong baroclinic deformation of tropospheric jet streams near the east coast of the United States appeared to radiate inertia gravity waves into the stratosphere. On plotting radiance perturbations in all 30 beams as horizontal "pushbroom" images, two-dimensional wavelike oscillations were revealed with impressively coherent linear phase lines in the horizontal. These structures were seen in different AMSU-A stratospheric channels, extending from the lower to the upper stratosphere. Their results appear to show AMSU-A "imaging" the horizontal structure of a jet-generated gravity wave packet as it propagates through the stratosphere. Such measurements, if validated, could provide a valuable new global measurement capability for stratospheric gravity waves, since to date only some very limited $4.3 \mu \mathrm{m}$ Midcourse Space Experiment (MSX) radiances have horizontally imaged stratospheric gravity waves from orbit (Dewan et al., 1998).

Experience to date has shown that a detailed understanding of the visibility characteristics of a satellite instrument to gravity waves is critical to a proper interpretation of waveinduced perturbations in the acquired data (Alexander, 1998; McLandress et al., 2000; Preusse et al., 2000; Jiang et al., 2004; Wu et al., 2006). How gravity waves might manifest in stratospheric radiances acquired by AMSU-A is not well understood at present. Thus, in Sect. 3 we develop a simple forward model of the in-orbit AMSU-A Channel 9 radiance acquisition, aimed at understanding how three-dimensional gravity wave oscillations might appear in these radiances. While we take care to validate the accuracy of the model 
we develop, we also seek relevant simplifications whereever possible so that we can gain physical insight into how gravity waves are observed, and can also apply the model more easily to interpret future AMSU-A observations. We quantify the sensitivity of Channel 9 radiances to gravity waves of different vertical and horizontal wavelengths via spectral analysis of the resulting weighting functions (Sect. 4) and three-dimensional forward modeling using idealized threedimensional gravity wave temperature oscillations (Sect. 5). An observational study of a gravity wave event imaged in AMSU-A radiances that utilizes and validates the modeling predictions from this work forms the subject of a companion paper (Eckermann et al., 2006b).

\section{Advanced Microwave Sounding Unit-A}

The Advanced Microwave Sounding Unit (AMSU) is a cross-scanning passive microwave sounding instrument currently deployed on five different satellites: the NOAA-15, NOAA-16, NOAA-17 and NOAA-18 meteorological satellites (Mo, 1996; Kidder et al., 2000), and NASA's Earth Observation System (EOS) Aqua satellite (Lambrigtsen, 2003).

AMSU consists of three separate hardware modules, denoted AMSU-A1, AMSU-A2, and AMSU-B. AMSU-B is known as the Humidity Sounder for Brazil (HSB) on EOS Aqua, and was superseded by the Microwave Humidity Sounder (MHS) on NOAA-18: we do not use data from these modules in this study. AMSU-A1 has 12 channels sampling thermal oxygen emissions in the $50-58 \mathrm{GHz}$ band, and one channel at $89 \mathrm{GHz}$, while AMSU-A2 has two water vapor channels at 23.8 and $31.4 \mathrm{GHz}$ : together, these two modules comprise AMSU-A. The two AMSU-A2 channels are denoted AMSU-A channels 1 and 2. AMSU-A channels 3-8 are the first six AMSU-A1 channels centered at 50.3, $52.8,53.596 \pm 0.115,54.4,54.94$ and $55.5 \mathrm{GHz}$, respectively, with channel 5 sampling two $\mathrm{O}_{2}$ wing emission lines either side of the central line. Channels 9-14 sample $\mathrm{O}_{2}$ wing line emissions centered at $57.290 \mathrm{GHz}$, and channel 15 samples emissions at $89 \mathrm{GHz}$. AMSU-A channels $1-3$ and 15 are "window" channels that mostly sense surface parameters. The other 11 channels (4-14) are atmospheric temperature sounding channels that acquire radiances from progressively higher in the atmosphere, from $\sim 900 \mathrm{hPa}$ for channel 4 , up to $\sim 2.5 \mathrm{hPa}$ for channel 14 . Channels $4-8$ peak in the troposphere, while channels $9-14$ are stratospheric. For further details, see Table 1 and Fig. 3 of Goldberg et al. (2001).

Figure 1 depicts the AMSU-A scanning pattern with respect to the orbital geometry. The satellite, depicted by the square on the $\mathrm{Z}$ axis at its orbit altitude $Z_{\text {sat }}$, orbits along the $\mathrm{X}$ axis, tracing out a ground track at $Y=0$. The NOAA satellites orbit the Earth every $102 \mathrm{~min}$ at an altitude $Z_{\mathrm{sat}}=833 \mathrm{~km}$, and so move along the $X$ axis at $+7.4 \mathrm{~km} \mathrm{~s}^{-1}$. EOS Aqua orbits at a somewhat lower alti-

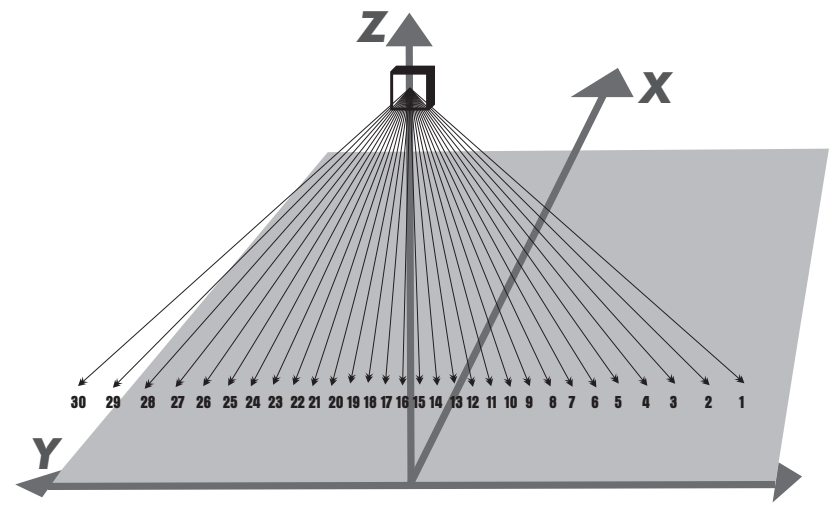

Fig. 1. Schematic depiction of the AMSU-A measurements. The satellite is depicted by the square on the $\mathrm{Z}$ axis and orbits along the $\mathrm{X}$ axis. From this platform, AMSU-A performs $j=1$...30 sequential step-and-stare measurements at equispaced off-nadir cross-track scan angles $\beta_{j}$, ranging from $\beta_{1}=-48.33^{\circ}$ to $\beta_{30}=+48.33^{\circ}$. Sign convention for quantities on the $\mathrm{Y}$ axis was chosen to preserve a right-handed coordinate system $(X, Y, Z)$.

tude of $Z_{\mathrm{sat}}=705 \mathrm{~km}$ and has an orbital period of $98.8 \mathrm{~min}$ (Parkinson, 2003), yielding a $7.5 \mathrm{~km} \mathrm{~s}^{-1}$ satellite velocity.

AMSU-A performs periodic cross-track scans along the $\mathrm{Y}$ axis. As depicted in Fig. 1, each scan cycle consists of 30 individual "step and stare" measurements at 30 different beam positions $j$ defined by their different equispaced offnadir cross-track viewing angles $\beta_{j}$ with respect to the subsatellite point, given by

$\beta_{j}=\frac{-155+10 j}{3}$ deg. $(j=1 \ldots 30)$.

Thus each scan starts at beam position $j=1$ $\left(\beta_{1}=-48.33^{\circ}\right)$, then progresses along $Y$ in $3.33^{\circ}$ steps to a final measurement at beam position $j=30\left(\beta_{30}=+48.33^{\circ}\right)$ : see also Fig. 2. The scanning pattern is symmetric about the subsatellite ground track $(Y=0)$. Each staring measurement lasts for $0.165 \mathrm{~s}$ followed by $\sim 37.5 \mathrm{~ms}$ to rotate to the next beam position, yielding a measurement every $\sim 0.2025 \mathrm{~s}$ and $\sim 6 \mathrm{~s}$ to perform a complete cross-track scan of 30 measurements. After each such scan cycle, $\sim 2 \mathrm{~s}$ is devoted to radiometric recalibration via viewing of an internal blackbody source and cold space, so that the total scan cycle period is $8 \mathrm{~s}$. For further details, see Lambrigtsen (2003).

\section{Simple forward model of AMSU-A Channel 9 radi- ance acquisition}

\subsection{Absorption}

Wu (2004) presented a simple model of the AMSU-A radiance measurement, which we develop further here. We focus here on the lowest-altitude stratospheric channel (Channel 9), which measures the central $57.290 \mathrm{GHz} \mathrm{\textrm {O } _ { 2 }}$ wing line 


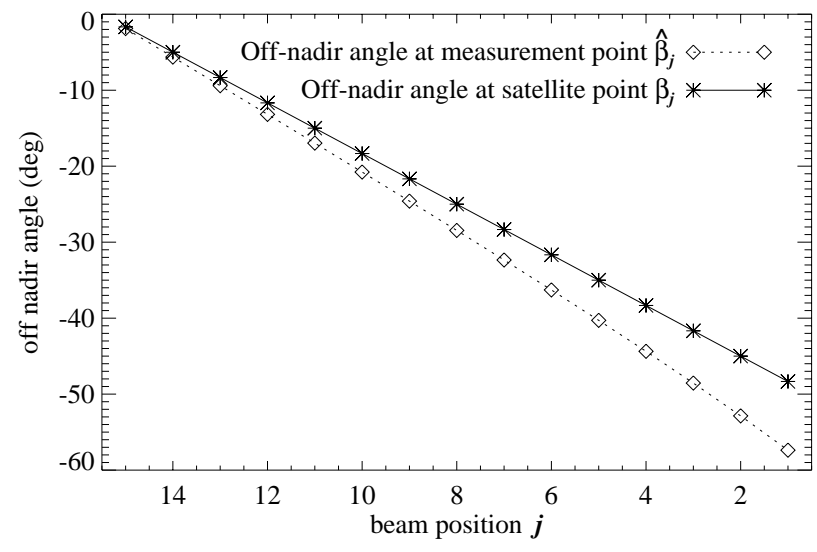

Fig. 2. Asterisks connected with solid line show the off-nadir crosstrack scan angles $\beta_{j}$ of AMSU-A beam positions $j=1-15$ depicted in Fig. 1. Diamonds connected with dotted line show the corresponding off-nadir angles $\hat{\beta}_{j}$ of the line-of-sight ray to the satellite at the "measurement point," defined as the point of peak response in the 3-D weighting functions (see later). These values are derived from Eq. (A2) in Appendix A for a nominal measurement altitude of $Z_{C}=18 \mathrm{~km}$. These $\hat{\beta}_{j}$ angles differ slightly from $\beta_{j}$ at the farthest off-nadir scan angles due to Earth curvature: see Eq. (A2).

emission. Initially, we will assume that this emission peaks low enough in the atmosphere $(\sim 60-90 \mathrm{hPa})$ that pressurebroadening dominates, so that it can be modeled to a good approximation as a single Lorentz line. Then, the absorption coefficient is the Lorentz-line solution for a gas with constant mixing ratio: $\tilde{k}_{v}=B p$, where $p$ is atmospheric pressure and $B$ is a line constant (Houghton et al., 1984). Radiance absorption along a ray path distance $s$ from the Earth's surface is then given by

$$
\begin{aligned}
\tau(s) & =\int_{0}^{s} B p\left(s^{\prime}\right) \rho_{\mathrm{O} 2}\left(s^{\prime}\right) d s^{\prime}=\int_{0}^{s} \frac{-B q_{\mathrm{O} 2} p^{2}\left(s^{\prime}\right)}{g H\left(s^{\prime}\right)} d s^{\prime} \\
& =\int_{0}^{s} \frac{A p^{2}\left(s^{\prime}\right)}{H\left(s^{\prime}\right)} d s^{\prime}=\int_{0}^{s} \frac{d \tau\left(s^{\prime}\right)}{d s} d s^{\prime},
\end{aligned}
$$

where $\rho_{\mathrm{O} 2}$ is the $\mathrm{O}_{2}$ mass density, $q_{\mathrm{O} 2}$ is the (constant) $\mathrm{O}_{2}$ mass mixing ratio, and $H$ is pressure scale height (Houghton et al., 1984).

\subsection{Antenna pattern}

Since the central AMSU-A antenna beam has an approximately Gaussian-shaped polar diagram (Lambrigtsen, 2003), we approximate the polar diagram along the cross track scan axis $\mathrm{Y}$ at beam position $j$ as

$$
\begin{aligned}
P_{1-D}\left(\beta, \beta_{j}\right) & \propto \exp \left\{-\left[\frac{\beta-\beta_{j}}{\beta_{W}}\right]^{2}\right\}, \\
\beta_{W} & =\frac{\beta_{\mathrm{HPBW}}}{2(\ln 2)^{1 / 2}},
\end{aligned}
$$

where $\beta$ is the off-nadir angle along the cross-track $Y$ direction, and the Gaussian antenna width $\beta_{W}$ is related via Eq. (4) to the quoted full-width half-power $(3 \mathrm{~dB})$ beamwidth of the AMSU-A1 antenna $\beta_{\mathrm{HPBW}}$, which is nominally $3.3^{\circ}$ but was measured by Mo (1996) to be $\sim 3.51^{\circ}$ for Channel 9 . We use this latter value in our model. Since the antenna pattern is symmetric in the along-track and cross-track directions (Mo, 1999), our two-dimensional antenna pattern is

$P_{2-D}\left(\alpha, \beta, \beta_{j}\right) \propto P_{1-D}\left(\beta, \beta_{j}\right) P_{1-D}(\alpha, 0)$,

where $\alpha$ is the off-nadir angle in the along-track $X$ direction: note that $\alpha_{j}=0$ for all $j$ in Eq. (5) since AMSU-A scans cross-track only (see Fig. 1).

\subsection{Radiative transfer model}

We adopt a simplified model of microwave radiative transfer in the $(X, Y, Z)$ plane that ignores noise, cloud scattering and surface effects, and invokes the Rayleigh-Jeans approximation to the microwave Planck function (e.g., Staelin, 1977). The brightness temperature (radiance) contribution for beam position $j$ at a distance $s$ from the surface along a ray path directed to the satellite is quantified by the gradient term

$$
\begin{aligned}
\frac{d T_{B}}{d s}\left(s, \beta_{j}\right) \propto & \int_{-\pi / 2}^{+\pi / 2} \int_{-\pi / 2}^{+\pi / 2} P_{2-D}\left(\alpha, \beta, \beta_{j}\right) \frac{d \tau(s)}{d s} \\
& T\left(X^{\prime}, Y^{\prime}, Z^{\prime}\right) e^{-\tau(s)} d \alpha d \beta,
\end{aligned}
$$

where $T(X, Y, Z)$ is the atmospheric temperature and $X^{\prime}(s, \alpha, \beta), Y^{\prime}(s, \alpha, \beta), Z^{\prime}(s, \alpha, \beta)$ are the positions in the atmosphere in the $(X, Y, Z)$ frame in Fig. 1 implied by geometry for a line-of-sight ray to the satellite inclined at off-nadir angles $\alpha$ and $\beta$. The total brightness temperature acquired by the instrument at beam position $j$ is

$T_{B}\left(\beta_{j}\right)=\int_{0}^{s_{\mathrm{sat}}} \frac{d T_{B}}{d s}\left(s^{\prime}, \beta_{j}\right) d s^{\prime}$.

where $s_{\text {sat }}$ is the total length of this ray path from the ground to the satellite.

Our model solves this simplified radiative transfer numerically in the $Y-Z$ plane by integrating along successive ray paths $s$ for a full range of angles $\beta$ centered about the central antenna beam $\beta_{j}$ for $\alpha=0$. These calculations use a full spherical geometry outlined briefly in Appendix A: see Fig. 16.

\subsection{Weighting functions}

For a purely vertical mean temperature profile $T(Z)$, our numerical model calculations in Sect. 3.3 yield the two-dimensional (2-D) temperature weighting functions $W_{j}(Y, Z)$ and then the one-dimensional (1-D) vertical weighting functions $W_{j}(Z)=\int_{-\infty}^{\infty} W_{j}(Y, Z) d Y$ for each beam position $j$. Since our radiative transfer model is idealized, to simplify the calculations further we used $H=7.5 \mathrm{~km}$ 
in Eq. (2), then adjusted the absorption constant $A$ in Eq. (2) to give best agreement with the Channel 9 1-D vertical weighting functions for the near-nadir beam positions $j=15-$ 16 published by Goldberg et al. (2001), based on rigorous radiative transfer calculations. We used the 1976 U.S. Standard Atmosphere temperature profile for $T(Z)$, although under these model approximations our final weighting functions have no explicit dependence on the temperature profile used.

\subsubsection{1-D vertical weighting functions}

The lower dotted curve in Fig. 3 shows the vertical weighting function $W_{j}(Z)$ for the near-nadir beams $(j=15-16)$ from our simple Lorentz-line model with a fitted constant $A$. It reproduces the Goldberg et al. (2001) profile (gray solid curve) quite well at altitudes near the peak response. In fact these near-nadir model weighting functions have shapes similar to the idealized analytical profile solutions $\propto p^{2} \exp \left[-\tilde{A} p^{2}\right]$ appropriate for a single Lorentz line emission from a gas with constant mixing ratio viewed in the nadir without antenna spreading (Houghton et al., 1984; Grody, 1993), where $\tilde{A}$ is a constant related to $A$.

This same constant $A$ value is then used in our model to evaluate all the other 1-D and 2-D weighting functions at all beam positions $j=1 \ldots 30$. The second (upper) dotted curve in Fig. 3 shows the resulting model-generated 1-D vertical weighting function at the largest off-nadir beam positions $j=1$ and $j=30$. The agreement with the corresponding profile of Goldberg et al. (2001) is again quite good given the simplicitly of our model.

Nonetheless, the model results diverge from the Goldberg et al. (2001) profiles most noticeably at the tails of the weighting functions at altitudes away from the peak response. Though these differences seem minor given the small values of the weighting functions here, simple 1$\mathrm{D}$ modeling calculations using sinusoidal vertical temperature perturbations revealed significant differences in acquired brightness temperature perturbation amplitudes between these profiles and the Goldberg et al. (2001) profiles for shorter vertical wavelength perturbations.

To obtain better fits, we tuned our absorption coefficient $A$ in Eq. (2) to have a pressure-altitude dependence $A(Z)$ that parameterizes the net effects of non-Lorentzian and/or overlapping lines (following, e.g., Waters et al., 1975; Poon, 1980; Grody, 1993). Closer fits were obtained by reducing $A$ with pressure altitude approximately linearly below $20 \mathrm{~km}$ and above $25 \mathrm{~km}$. Solid curves in Fig. 3 show the vertical weighting functions produced by these "tuned" Lorentz model runs. Since they fit the Goldberg et al. (2001) profiles better at all altitudes, we use weighting functions from this tuned model in all subsequent analysis.

The off-nadir $W_{j}(Z)$ curves in Fig. 3 peak higher in altitude since they traverse longer pathlengths through the atmosphere: the so-called "limb effect" (e.g., Grody, 1993). Note

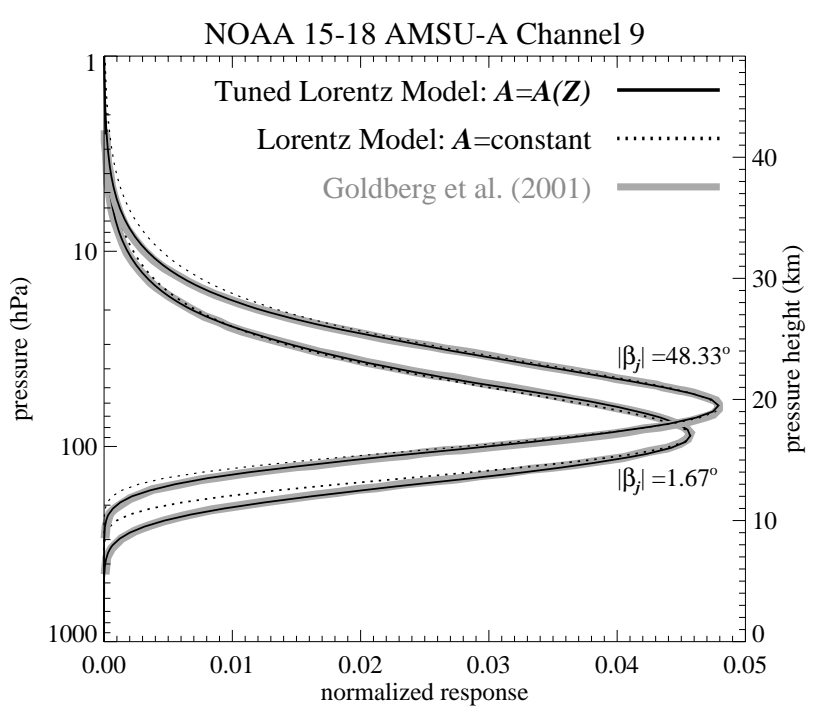

Fig. 3. Channel 91 -D vertical temperature weighting functions $W_{j}(Z)$ for the smallest off-nadir angle of $\beta_{j}= \pm 1.67^{\circ}$ and largest off-nadir angle of $\beta_{j}= \pm 48.33^{\circ}$, derived from our simple Lorentz model (constant $A$; dotted cuve) and tuned Lorentz model (heightvarying $A$, solid curve). Gray curves show results of Goldberg et al. (2001) using a complete radiative transfer model.

also that the off-nadir Channel 9 weighting function is narrower vertically and thus has a larger maximum value. This indicates that, for infinitely long horizontal wavelengths, offnadir beams should be slightly more sensitive to vertical temperature oscillations than the near-nadir beams.

\subsubsection{2-D and 3-D weighting functions}

Figure 4 profiles $W_{j}(Y, Z)$ from our tuned Lorentz model for AMSU-A measurements from the NOAA satellites at all 15 different off-nadir scan angles. The results illustrate not only an increase in the peak altitude of the weighting function with increasing $\left|\beta_{j}\right|$ due to the limb effect, but also an increasing cross-track width. These features are studied further in Sect. 3.5.

Since AMSU-A does not scan along-track, we assume no significant limb effect along-track, so that the along-track variations in the weighting functions are controlled solely by the antenna pattern. This yields a separable form for our assumed three-dimensional (3-D) AMSU-A weighting function

$W_{j}(X, Y, Z)=G(X) W_{j}(Y, Z)$,

where $G(X)$ specifies the along-track spreading at a given $Y, Z$ and beam position $j$ controlled by the antenna pattern Eq. (5) and spherical geometry. There will be a small additional smearing contribution along the $X$ direction due to the $7.4 \mathrm{~km} \mathrm{~s}^{-1}$ motion of the satellite and the $0.165 \mathrm{~s}$ staring 


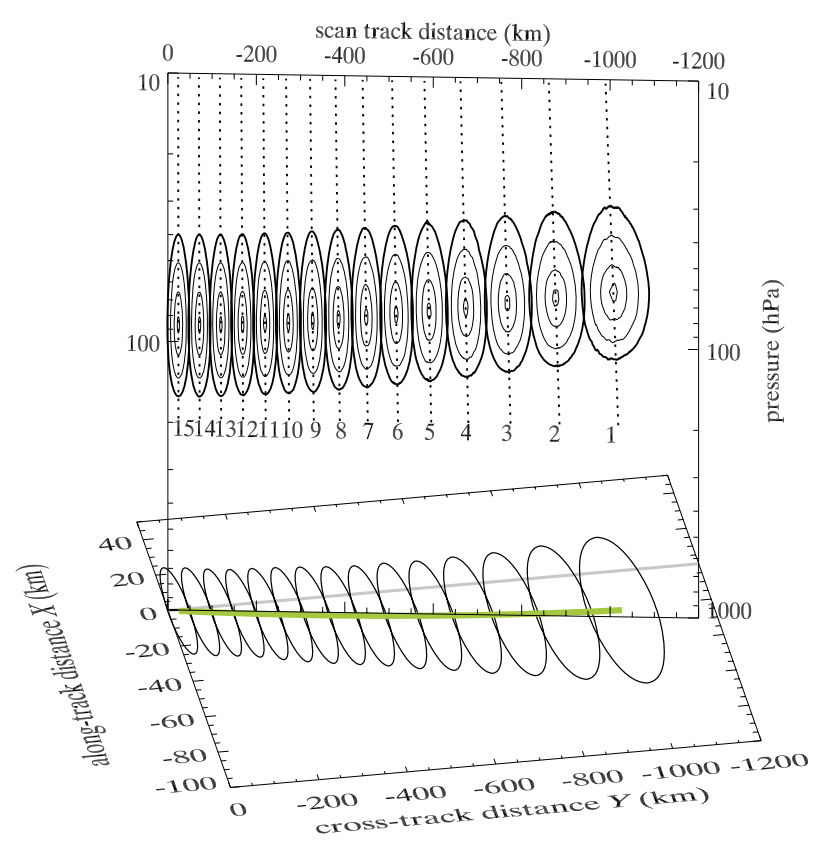

Fig. 4. Modeled AMSU-A Channel 9 weighting functions $W_{j}(Y, Z)$ for beam positions $j=1 \ldots 15$. Thick contours show the half-power $(50 \%)$ levels, the outer ranges of which are projected to the surface to depict the horizontal footprints. Other contours show the $70 \%, 90 \%$ and $99 \%$ levels. Dotted lines show line of sight ray paths from the peaks in each weighting function to the satellite point, taking into account Earth curvature: the off nadir angles $\hat{\beta}_{j}$ that each of these ray paths makes at the weighting function peak are plotted as the dotted curve in Fig. 2. The $W_{j}(Y, Z)$ contours are plotted along a vertical plane aligned along an approximate scan axis that is offset slightly from the cross-track axis $\mathrm{Y}$ due to the $7.4 \mathrm{~km} \mathrm{~s}^{-1}$ motion of the satellite along the $X$ axis during the $3 \mathrm{~s}$ taken to scan through beam positions $j=1-15$. The actual $X-Y$ scan track is plotted in green, and curves slightly in the $X-Y$ plane. Note the different $\mathrm{X}$ and $\mathrm{Y}$ axis scales: footprint diameters along track are in fact narrower than those cross track (see Fig. 5).

time, but the effect is small in comparison to $G(X)$ and so is ignored hereafter.

\subsection{Horizontal footprints}

The half-power levels for $W_{j}(Y, Z)$ (thick contours in Fig. 4) are used to specify the cross-track diameters of the measurement "footprints" $\left(f_{Y}\right)_{j}$. Using Eq. (8), and given the symmetric antenna pattern of Eqs. (3)-(4), we can convert these $\left(f_{Y}\right)_{j}$ values from our model into associated alongtrack half-power footprint widths $\left(f_{X}\right)_{j}$ via approximate geometrical relations linking the two for a curved Earth: see Appendix A. The result is a scaling ratio

$$
\frac{\left(f_{X}\right)_{j}}{\left(f_{Y}\right)_{j}}=\frac{2 \sin \phi\left(\beta_{j}\right) \tan \left[\frac{\beta_{\mathrm{HPBW}}}{2}\right]}{\sin \beta_{j}\left[\phi\left(\beta_{j}+\frac{\beta_{\mathrm{HPBW}}}{2}\right)-\phi\left(\beta_{j}-\frac{\beta_{\mathrm{HPBW}}}{2}\right)\right]},
$$

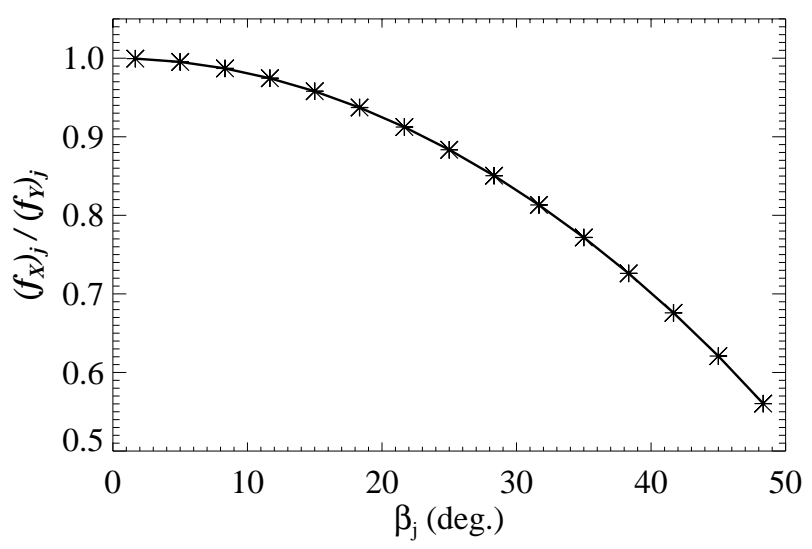

Fig. 5. Ratio of along-track to cross-track footprint diameters $\left(f_{X}\right)_{j} /\left(f_{Y}\right)_{j}$ versus scan angle $\beta_{j}$ based on Eqs. (9) and (10) for NOAA satellite orbit parameters and a nominal Channel 9 measurement altitude of $Z_{C}=18 \mathrm{~km}$.

where

$\phi(\beta)=\arcsin \left[\frac{\left(R_{E}+Z_{\mathrm{sat}}\right) \sin \beta}{R_{E}+Z_{C}}\right]-\beta$,

is the angle from the center of the Earth between the satellite and the measurement point for a given cross-track off-nadir angle $\beta, R_{E}$ is Earth radius and $Z_{C}$ is the altitude of the measurement point. Given $\left(f_{Y}\right)_{j}$ from our model, we derive $\left(f_{X}\right)_{j}$ using Eqs. (9) and (10). Ratios from Eq. (9) are plotted in Fig. 5 for NOAA satellite orbit parameters. The elliptical half-power horizontal footprints implied by $\left(f_{X}\right)_{j}$ and $\left(f_{Y}\right)_{j}$ are projected onto the $Z=0$ surface in Fig. 4. Figure 6a shows how the $7.4 \mathrm{~km} \mathrm{~s}^{-1}$ velocity of the NOAA satellites along the $\mathrm{X}$ axis maps these observational footprints into two-dimensional horizontal measurement coverage as the instrument cyclically scans cross-track. Almost all regions at cross-track distances $\pm 1100 \mathrm{~km}$ either side of the satellite ground track are measured with $>50 \%$ sensitivity. Footprint sizes increase at the outer scan angles and overlap (oversample) in the $X$ direction. These footprints, derived from our tuned Channel 9 radiance acquisition model, compare well with those from more detailed calculations of surface footprints for AMSU-A window channels (see, e.g., Fig. 1 of Kidder et al., 2000).

Figure $6 \mathrm{~b}$ shows the corresponding footprints calculated for the AMSU-A on EOS Aqua. The lower orbit altitude ( $Z_{\mathrm{sat}}=705 \mathrm{~km}$ compared to $Z_{\mathrm{sat}}=833 \mathrm{~km}$ for the NOAA satellites) produces correspondingly smaller footprints and less total horizontal coverage cross track. Since the satellite velocity is about the same, these smaller footprints mean that not all regions within the swath are sampled at $>50 \%$ sensitivity from EOS Aqua. 
(a) NOAA 15-18 Channel 9 AMSU-A Footprints

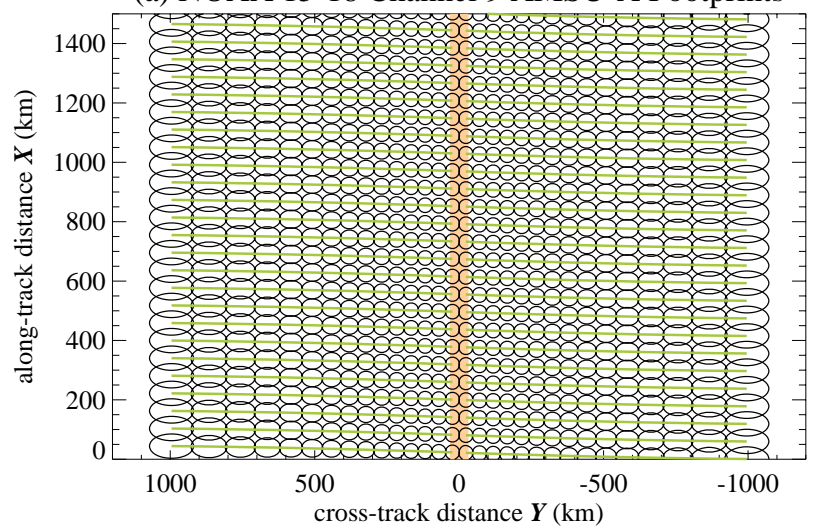

(b) EOS Aqua Channel 9 AMSU-A Footprints

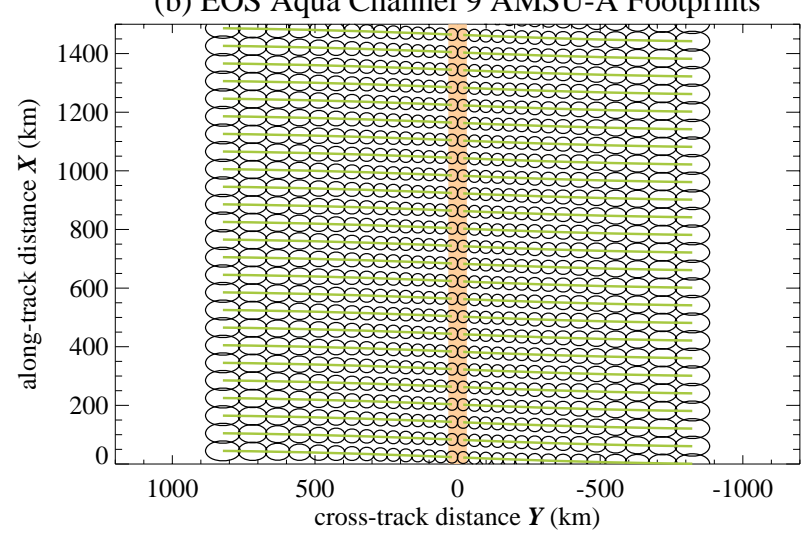

Fig. 6. AMSU-A Channel 9 horizontal footprints as a function of along-track and cross-track distances traced out by the AMSU-A scanning pattern from (a) NOAA-15 through NOAA-18 satellites and (b) EOS Aqua. Orange line shows the satellite ground track. Green curves show the scanning pattern from right-to-left across $Y$ as the satellite moves along $X$.

\subsection{Simulated brightness temperature measurement}

Having derived weighting functions, we now use them to simulate radiance acquisition in the presence of varying atmospheric temperatures. If we center the peak sensitivity in the various 3-D weighting functions $W_{j}(X, Y, Z)$ at $X=Y=0$ and define $X_{j}, Y_{j}$ to be the current central position of this beam's horizontal measurement footprint, then the acquired brightness temperature, Eq. (7), can be reexpressed as

$$
\begin{gathered}
T_{B}\left(X_{j}, Y_{j}\right)=\int_{-\infty}^{\infty} \int_{-\infty}^{\infty} \int_{0}^{Z_{\text {sat }}} W_{j}\left(X-X_{j}, Y-Y_{j}, Z\right) \\
T(X, Y, Z) d X d Y d Z
\end{gathered}
$$

where $T(X, Y, Z)$ is atmospheric temperature and here the 3 -D weighting functions are normalized such that

$\int_{-\infty}^{\infty} \int_{-\infty}^{\infty} \int_{0}^{Z_{\text {sat }}} W_{j}(X, Y, Z) d X d Y d Z=1$
We evaluate Eq. (11) numerically in Fig. 7 in simulating an AMSU-A measurement from NOAA satellites of the mean vertical temperature profile over southern Scandinavia on 14 January 2003 at 12:00 UTC, as specified by analysis fields in Fig. 7a from the European Center for Medium Range Weather Forecasts (ECMWF). Figure 7b plots the simulated Channel 9 brightness temperatures, and Fig. 7c plots the mean brightness temperatures as a function of scan angle $\beta_{j}$.

We see a decrease in the Channel 9 brightness temperature with increasing $\left|\beta_{j}\right|$, caused by the increase in height of the peak sensitivity of the weighting functions with increasing $\left|\beta_{j}\right|$ (Figs. 3-4) coupled with the decrease with height in temperatures in Fig. $7 \mathrm{a}$ in the $\sim 60-90 \mathrm{hPa}$ region where Channel 9 measurement sensitivities peak (Fig. 3). This simulates the well-known "limb darkening" effect typically observed in raw swath-scanned microwave radiance imagery from the troposphere due to negative tropospheric lapse rates (Kidder and Vonder Haar, 1995): such effects are corrected/adjusted when retrieving temperatures from these data (Goldberg et al., 2001). Figure 7c reveals a fairly smooth cross-track trend in the limb darkening with increasing $\left|\beta_{j}\right|$, consistent with the observational study of $\mathrm{Wu}$ (2004), who found that crosstrack trends in raw AMSU-A brightness temperatures due to limb effects could be accurately removed by fitting a leastsquares polynomial to the cross-track radiances.

\section{Spectral sensitivity to gravity waves}

From Eq. (11) we see that (to within a sign and vertical shift convention) the 3-D AMSU-A weighting functions are convolved through 3-D atmospheric temperature fields by the cyclical scanning pattern to yield a final horizontal map of brightness temperatures. Since convolution corresponds to multiplication in the Fourier domain, then the Fourier Transforms of the weighting functions $W_{j}(X, Y, Z)$ define the spectral visibility (Jiang et al., 2004) of AMSU-A radiances to sinusoidal temperature structure with different horizontal and vertical wavelengths.

4.1 Fourier Transforms of two-dimensional weighting functions

Figure 8 plots the Fourier Transforms $\hat{W}_{j}\left(k_{Y}, k_{Z}\right)$ of the NOAA 15-18 AMSU-A weighting functions $W_{j}(Y, Z)$ at a sequence of beam positions, ranging from far off-nadir (panel a, $j=2, \beta_{j}=-45^{\circ}$ ) to near-nadir (panel d, $j=15$, $\left.\beta_{j}=-1.67^{\circ}\right)$. For a given gravity wave of wavenumber $\left(k_{Y}, k_{Z}\right)$, these 2-D spectra define visibilities at both the beam position $j$ and its conjugate beam position $j_{*}=31-j$ (such that $\beta_{j_{*}}=-\beta_{j}$ ), since

$\hat{W}_{j_{*}}\left(k_{Y}, k_{Z}\right)=\hat{W}_{j}\left(-k_{Y}, k_{Z}\right)$.

Equation (13) arises due to the symmetry of the AMSU-A scanning pattern about the subsatellite point (ignoring small 

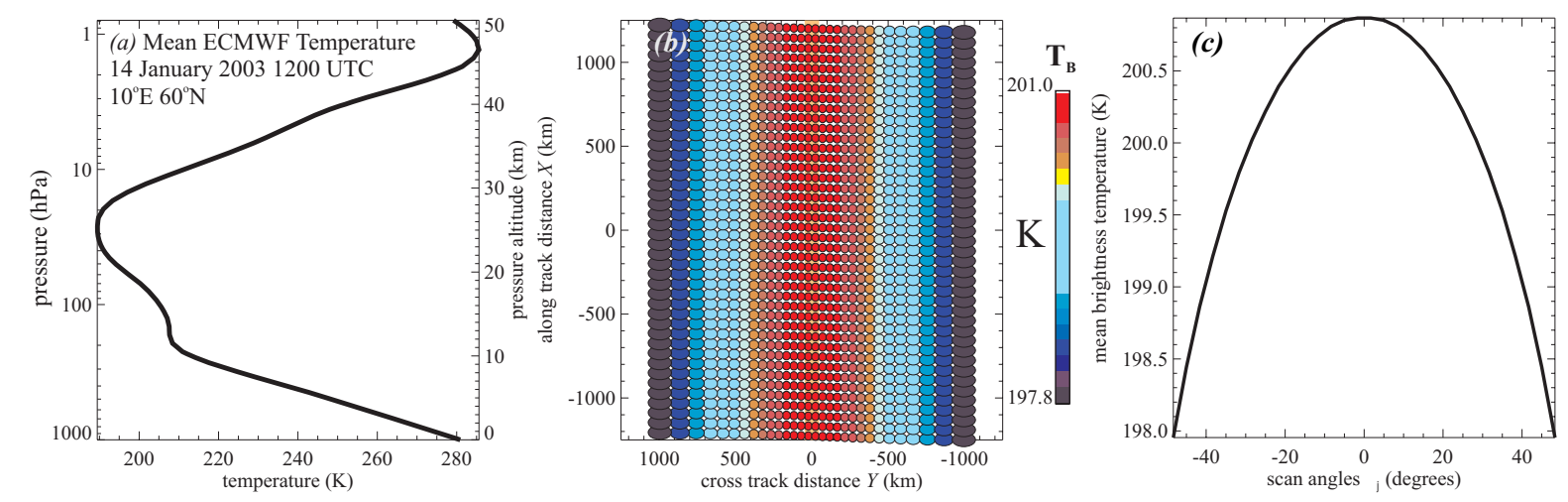

Fig. 7. (a) Mean temperature profile from the ECMWF analysis for 14 January 2003 at 12:00 UTC at $10^{\circ} \mathrm{E}, 60^{\circ} \mathrm{N}$; (b) model simulation of the AMSU-A Channel 9 brightness temperatures $T_{B}\left(X_{j}, Y_{j}\right)$ obtained from sampling this temperature profile using Eq. (11); (c) mean brightness temperatures from (b) as a function of $\beta_{j}$.

asymmetries introduced by the satellite motion: see Fig. 6). Physically, Eq. (13) means that observing an infinite wave train of wavenumber $\left(k_{Y}, k_{Z}\right)$ at beam position $j_{*}$ is equivalent to observing the identical antipropagating wave train of wavenumber $\left(-k_{Y}, k_{Z}\right)$ at beam position $j$. Note also that $\hat{W}_{j}\left(k_{Y}, k_{Z}\right)=\hat{W}_{j}\left(-k_{Y},-k_{Z}\right)$ for all $k_{Y}, k_{Z}$ and $j$.

Since the near-nadir observations have much narrower cross-track footprint diameters $\left(f_{Y}\right)_{j}$ than the far off-nadir observations, they can resolve structure with much shorter cross-track wavelengths $\lambda_{Y}=2 \pi /\left|k_{Y}\right|$, as evidenced by the broader spectral sensitivity along the $k_{Y}$ axis in Fig. $8 \mathrm{~d}$ compared to Fig. 8a. The sensitivity to vertical wavelengths $\lambda_{Z}=2 \pi /\left|k_{Z}\right|$ is slightly better for the $j=2$ spectrum compared to the $j=15$ spectrum due to the vertical narrowing of the weighting function response due to the limb effect, noted earlier in Fig. 3.

Gravity wave detection requires a brightness temperature oscillation that lies above the noise floor, which is quantified for AMSU-A by the so-called noise equivalent delta temperature $(\mathrm{NE} \Delta \mathrm{T})$. An NE $\Delta \mathrm{T}=0.236 \mathrm{~K}$ was measured prior to launch for Channel 9 of the NOAA AMSU-A instruments (Mo, 1996). However, Wu (2004) used observed minima in AMSU-A brightness temperature variances on NOAA 15, 16 and 17 to infer a smaller Channel 9 r.m.s. noise value of 0.15 $0.16 \mathrm{~K}$, a value similar to the $0.16 \mathrm{~K} \mathrm{NE} \Delta \mathrm{T}$ measured prelaunch for Channel 9 of the AMSU-A instrument on Aqua (Lambrigtsen, 2003). Hence we will use $0.16 \mathrm{~K}$ as our Channel 9 noise floor.

While extensive averaging of radiance variances can yield reliable geophysical variance estimates near or even below nominal noise variances (Jiang et al., 2004; Wu, 2004), here we will assume measurement of a single wave during a single satellite overpass. A conservative detectability criterion for such an observation is a signal-to-noise ratio of $\gtrsim 2$, and thus brightness temperature fluctuations of $\pm 0.3 \mathrm{~K}$ or greater. While r.m.s. temperature amplitudes for gravity waves in the extratropical lower stratosphere are $\sim 1-3 \mathrm{~K}$ (e.g., Eckermann et al., 1995; Tsuda et al., 2000), specific wave events can have peak amplitudes as large as $10 \mathrm{~K}$ (e.g., Hertzog et al., 2002; Eckermann et al., 2006a). Choosing a peak gravity wave temperature amplitude $T_{\text {peak }}=3 \mathrm{~K}$ at the low end of this $1-10 \mathrm{~K}$ range, we require a minimum spectral visibility of 0.1 to detect this wave at our $\pm 0.3 \mathrm{~K}$ lower limit for brightness temperature. This nominal threshold for detectability is highlighted as the bold contour in each panel of Fig. 8.

Figure 9 plots the corresponding visibility spectra for the AMSU-A on EOS Aqua. The smaller footprints in Fig. 6b yield $\hat{W}_{j}\left(k_{Y}, k_{Z}\right)$ spectra that are horizontally elongated compared to the corresponding NOAA AMSU-A spectra in Fig. 8, highlighting the Aqua instrument's greater sensitivity to cross-track wavelengths $\lambda_{Y}$.

These 2-D visibility spectra at the mid-range scan angles show small but noticeable asymmetries about the $k_{Y}=k_{Z}=0$ axes, which indicate via Eq. (13) cross-track asymmetries in the response to gravity waves. These effects are investigated further in Sect. 4.3.

\subsection{Spectral response versus beam position for a given wave}

Next we use these $\hat{W}_{j}\left(k_{Y}, k_{Z}\right)$ spectra to investigate how the AMSU-A visibility to a 2-D temperature oscillation of a given $\lambda_{Y}$ and $\lambda_{Z}$ varies at each beam position $j$. Figure 10a plots visibilities estimated from numerical model spectra at $\lambda_{Y}=400 \mathrm{~km}$ and $\lambda_{Z}=12 \mathrm{~km}$. We set $k_{Y}$ positive and $k_{Z}$ negative, consistent with a gravity wave with an upward vertical group velocity propagating along the positive $\mathrm{Y}$ axis direction in Fig. 1.

We focus first on visibility variations for the negative scan angles $\beta_{j}$ in Fig. 10a $(j=1 \ldots 15)$. For the near-zenith beam $(j=15)$, the NOAA AMSU-A visibility is $\sim 13 \%$, then increases with increasing $\left|\beta_{j}\right|$ to a peak of $\sim 13.7 \%$ at $j \sim 6-$ 8. From $j=6$ to $j=1$, however, the response decreases with 


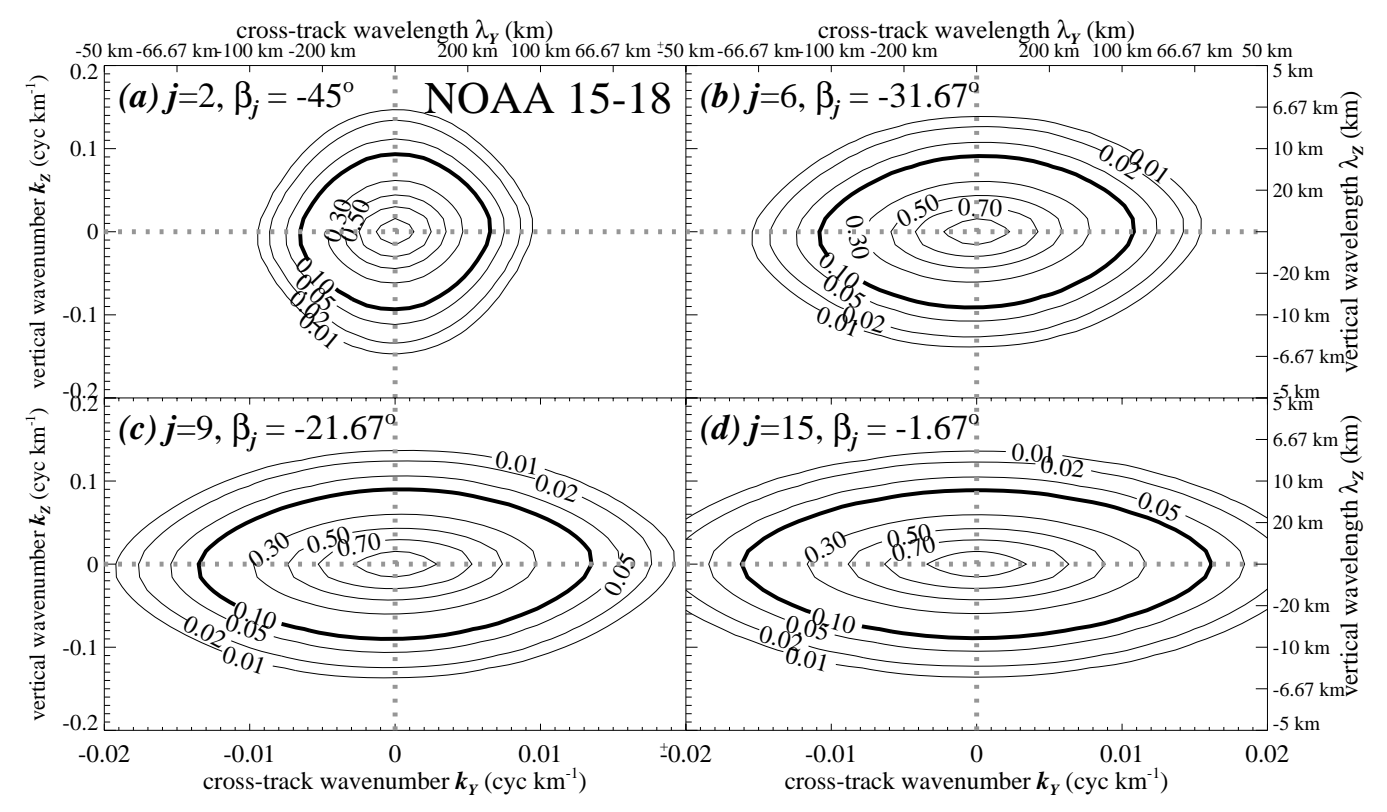

Fig. 8. Fourier Transforms $\hat{W}_{j}\left(k_{Y}, k_{Z}\right)$ computed from the model-generated 2-D Channel 9 weighting functions $W_{j}(Y, Z)$ for the AMSU-A on the NOAA $15-18$ satellites in Fig. 4 and normalized to a peak response of unity, for (a) $j=2\left(\beta_{2}=-45^{\circ}\right),\left(\right.$ b) $j=6\left(\beta_{6}=-31.67^{\circ}\right),($ c) $j=9$ $\left(\beta_{9}=-21.67^{\circ}\right)$, and (d) $j=15\left(\beta_{15}=-1.67^{\circ}\right)$.

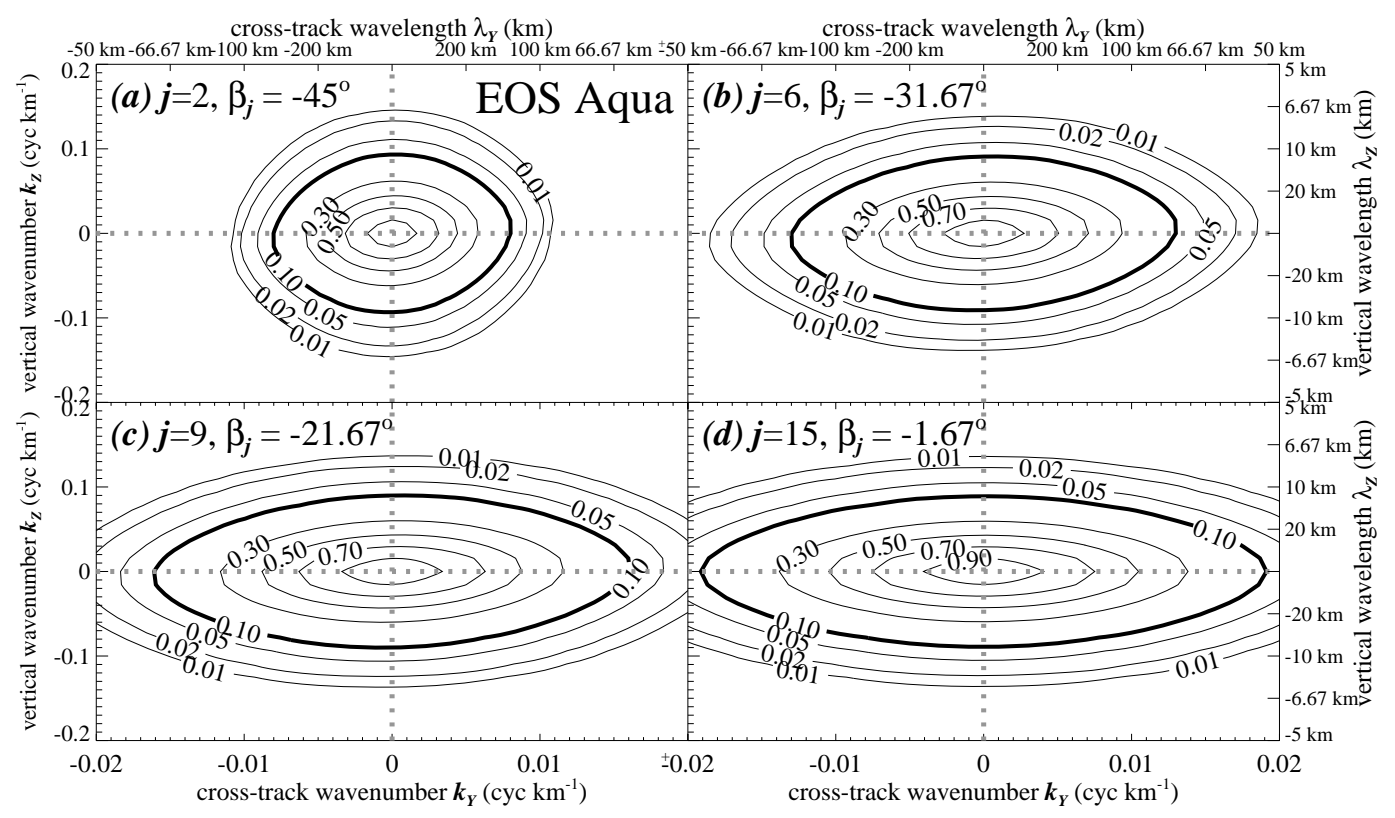

Fig. 9. As for Fig. 8, but showing Channel 9 model 2-D weighting function Fourier Transforms $\hat{W}_{j}\left(k_{Y}, k_{Z}\right)$ for the AMSU-A on EOS Aqua.

increasing $\left|\beta_{j}\right|$. This decreasing trend in visibility is due to the greater influence of cross-track footprint widths $\left(f_{Y}\right)_{j}$, which grow to diameters $\mathrm{O}(100 \mathrm{~km})$ at the outermost scan angles (see Fig. 6a) and thus significantly degrade detection of this $\lambda_{Y}=400 \mathrm{~km}$ wave. Note that the corresponding curve for the AMSU-A on EOS Aqua (dotted curve in Fig. 10a) shows larger visibilities at all $j$ and a peak visibility that occurs at a larger $\left|\beta_{j}\right|$ than for the NOAA AMSU-A curve. Both features are consistent with the smaller cross-track footprint sizes of the Aqua AMSU-A at each beam position (see Fig. 6). 

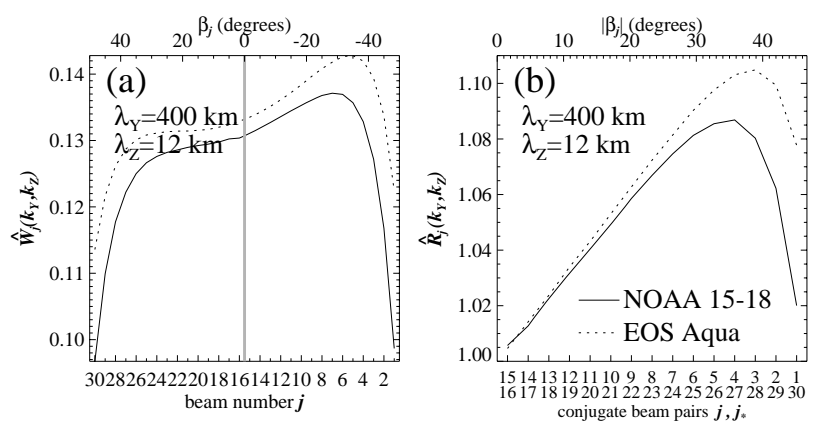

Fig. 10. (a) Spectral visibilities $\hat{W}_{j}\left(k_{Y}, k_{Z}\right)$ as a function of beam position $j$ for $\lambda_{Y}=400 \mathrm{~km}\left(k_{Y}>0\right)$ and $\lambda_{Z}=12 \mathrm{~km}\left(k_{Z}<0\right)$ for the AMSU-A on NOAA (solid curve) and EOS Aqua (dotted curve). (b) Ratio $\hat{R}_{j, j_{*}}\left(k_{Y}, k_{Z}\right)$ of the negative scan angle response to the positive scan angle response in (a) for each of the 15 conjugate beam pairs $j$ and $j_{*}$.

\subsection{Cross-track asymmetries in visibility}

Visibility curves at the positive scan angles $(j=16 \ldots 30)$ in Fig. 10a differ in both magnitude and shape to those just discussed at the negative scan angles $(j=1 \ldots 15)$. Figure $10 \mathrm{~b}$ plots the ratio of these visibilities for each of the 15 conjugate beam pairs $\left(j, j_{*}\right)$, defined by their identical off-nadir angles $\left|\beta_{j}\right|=\left|\beta_{j_{*}}\right|$. From Eq. (13), this ratio is given by

$\hat{R}_{j, j_{*}}\left(k_{Y}, k_{Z}\right)=\frac{\hat{W}_{j}\left(k_{Y}, k_{Z}\right)}{\hat{W}_{j_{*}}\left(k_{Y}, k_{Z}\right)}=\frac{\hat{W}_{j}\left(k_{Y}, k_{Z}\right)}{\hat{W}_{j}\left(-k_{Y}, k_{Z}\right)}$.

Asymmetry is introduced here by our wave structure and our sign choices for its wavenumbers $\left(k_{Y}>0, k_{Z}<0\right)$. To first order, a gravity wave is more visible to a scanning instrument when its phase lines roughly coalign with the tilt angle $\hat{\beta}_{j}$ of the line-of-sight ray from the measurement point to the satellite (e.g., Wu et al., 2006). It is straightforward to show (see Appendix A) that $\hat{\beta}_{j}=\beta_{j}+\phi\left(\beta_{j}\right)$ : these values are plotted as the dotted curve in Fig. 2. The phase lines of the wave considered in Fig. 10 are aligned at an off-nadir angle of $\arctan \left(k_{Z} / k_{Y}\right)=-88.28^{\circ}$. Thus this wave should be more visible at the negative scan angles than at the positive scan angles, consistent with visibility ratios $\hat{R}_{j, j_{*}}\left(k_{Y}, k_{Z}\right)$ greater than unity in Fig. 10b. This also explains (to first order) the increase in these ratios with increasing $\left|\beta_{j}\right|$ over the $j \sim 15-5$ range in Fig. 10b.

Interestingly, however, this increase in asymmetry with scan angle abates at $j \sim 5$ and the cross-track asymmetry then decreases with increasing $\left|\beta_{j}\right|$ at the outermost scan angles $(j=1 \ldots 5)$. This feature is somewhat surprising given that the outermost beam position pairs $\left(j, j_{*}\right)$ have the greatest differences in their respective scan angles $\beta_{j}$ and $\beta_{j_{*}}$, and thus might be expected to exhibit the largest cross-track asymmetries in their gravity wave visibilities. That they do not merits analysis.
In studying large cross-track asymmetries in the response of MLS saturated limb-track radiances to gravity waves, McLandress et al. (2000) and Jiang et al. (2004) introduced the concept of an effective line-of-sight angle, $\tilde{\beta}$. The combined effects of vertical variations in absorption and antenna spreading across the MLS viewing direction combined to yield a final 2-D MLS weighting function that was not symmetric about the line of sight direction $\hat{\beta}$, but was instead approximately symmetric about an effective line of sight direction $\tilde{\beta}$ that was more horizontally aligned than $\hat{\beta}$ (Wu and Waters, 1996a). Gaussian analytical approximations to the MLS vertical weighting functions and antenna pattern enabled McLandress et al. (2000) to evaluate $\tilde{\beta}$ analytically. Rotating the (Y,Z) axes to new tilted axes $\left(\mathrm{Y}^{\prime}, \mathrm{Z}^{\prime}\right)$, such that $\mathrm{Y}^{\prime}$ was aligned along this MLS effective line-of-sight direction $\tilde{\beta}$, led to a separable 2-D MLS weighting function with elliptical contours whose long axis was aligned along $\mathrm{Y}^{\prime}$ and whose short axis was aligned along $Z^{\prime}$. Peak responses occurred for gravity waves whose phase lines were aligned parallel to $\mathrm{Y}^{\prime}$, since their oscillations (orthogonal to phase lines along their wavenumber vector) occur along the shortest $Z^{\prime}$ axis of the weighting function and thus suffer the least smearing. Hence the term "effective line of sight" for this MLS Y' axis.

We can derive similar angles $\tilde{\beta}_{j}$ for the AMSU-A weighting functions, though the nomenclature "effective line of sight" is not apt here, since, unlike limb viewers like MLS, $\tilde{\beta}_{j}$ and $\hat{\beta}_{j}$ are very different for sublimb viewers like AMSUA. Hence we refer to $\tilde{\beta}_{j}$ as the cross-track tilt angle of the AMSU-A weighting function, and $Y_{j}^{\prime}$ as its cross-track tilt axis. Also, unlike the MLS case, here we cannot evaluate $\tilde{\beta}_{j}$ analytically since our AMSU-A weighting functions are numerical, nonGaussian in the vertical and somewhat asymmetric cross-track due to spherical effects. Nonetheless, we can estimate $\tilde{\beta}_{j}$ numerically as follows.

First, we note from Jiang et al. (2004) that their MLS effective line-of-sight axis $\mathrm{Y}^{\prime}$ can be defined approximately as the line interconnecting local maxima in vertical profiles of the 2-D weighting functions at different cross track positions $Y$ : see, e.g., their Fig. 8c. Thus, we estimate cross-track tilt angles $\tilde{\beta}_{j}$ by evaluating altitudes $\tilde{Z}$ of the local maxima in vertical profiles of $W_{j}(\tilde{Y}, Z)$ at a series of different $\tilde{Y}$ values. The resulting locus of $(\tilde{Y}, \tilde{Z})$ coordinates specifies the crosstrack tilt axis $\mathrm{Y}_{j}^{\prime}$ : the result of such a calculation for the $j=7$ Aqua AMSU-A 2-D weighting function is plotted as the gray thick solid line in Fig. 11a. The off-nadir tilt angle of this line is $\tilde{\beta}_{j}$. One can see from Fig. 11a that the width of the 2-D weighting functions along a $Z_{j}^{\prime}$ axis that lies orthogonal to this solid gray $\mathrm{Y}_{j}^{\prime}$ axis is narrower than the width along the dashed line-of-sight axis, for example. Thus, waves whose phase lines align parallel to $\mathrm{Y}_{j}^{\prime}$ should be more visible than those whose phases are aligned differently.

Figure $11 \mathrm{~b}$ plots our numerical estimates of tilt angles $\tilde{\beta}_{j}$ as a function of beam position $j$ for both the NOAA and 

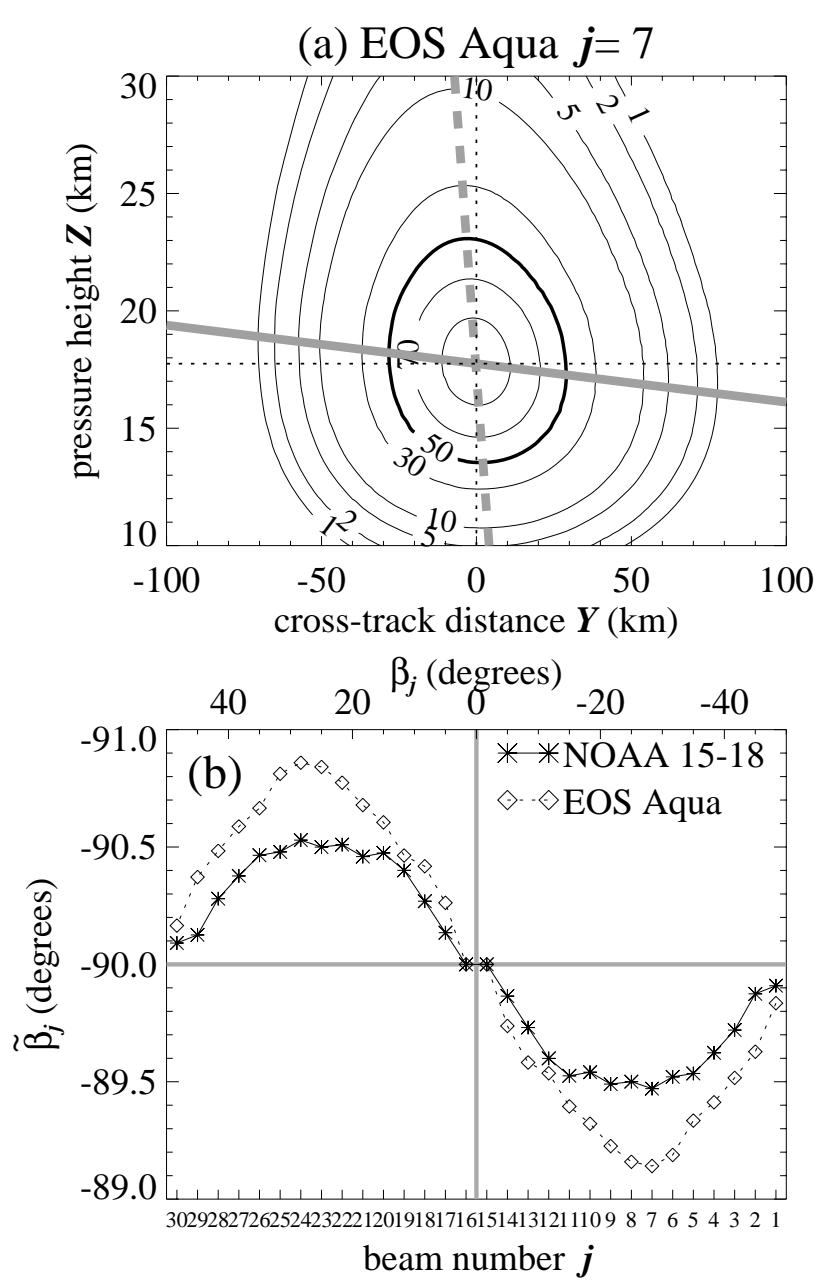

Fig. 11. (a) $W_{j}(Y, Z)$ at $j=7$ for $\mathrm{AMSU}-\mathrm{A}$ on EOS Aqua. Contours are values relative to the peak response, expressed as a percentage. The $50 \%$ contour is shown in bold. Dotted gray line shows the lineof-sight ray to the satellite. Solid gray curve connects local maxima in vertical cross sections through the weighting function at different $Y$, and specifies the cross-track tilt axis $\mathrm{Y}_{j}^{\prime}$. The off-nadir tilt angle of this gray line is $\tilde{\beta}_{j}$. (b) $\tilde{\beta}_{j}$ angles computed as in (a) for all the weighting functions $W_{j}(Y, Z)$ for both the NOAA (solid curve) and EOS Aqua (dotted curve) AMSU-A.

Aqua AMSU-A weighting functions. These angles are all close to $-90^{\circ}$ (i.e. very little axis tilt). On progressing outwards from nadir in Fig. 11b, these angles become progressively more tilted out to the mid-range scan angles, then level off and start to return towards more horizontal $\mathrm{Y}_{j}^{\prime}$ axes at the outermost scan angles. This return to symmetry at the outermost scan angles is due mostly to much wider horizontal footprint diameters $\left(f_{Y}\right)_{j}$ from antenna spreading. For example, the analytical expressions of McLandress et al. (2000) and Jiang et al. (2004) show that as the ratio of the widths of the antenna spreading to vertical absorption (their $\gamma^{-1 / 2}$ parameter) increases at the measurement point, the MLS effective line-of-sight axis $\mathrm{Y}^{\prime}$ gets pushed further towards the
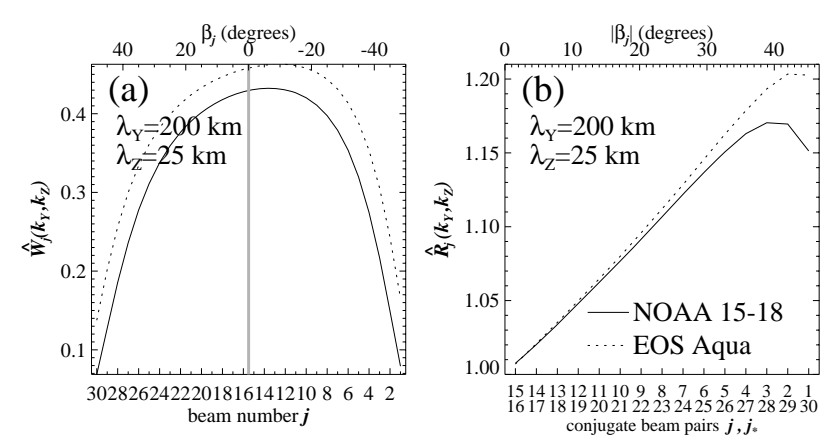

Fig. 12. As for Fig. 10, but for $\lambda_{Y}=200 \mathrm{~km}$ and $\lambda_{Z}=25 \mathrm{~km}$.

horizontal. This width ratio becomes large at the outermost AMSU-A scan angles since antenna spreading widths increase significantly here due to longer ray path lengths from the satellite to the measurement point. Thus the trend back to symmetric responses at far off-nadir scan angles in Fig. 10b is consistent with the transition to less-tilted $\mathrm{Y}_{j}^{\prime}$ axes for the weighting functions in Fig. $11 \mathrm{~b}$.

Figure 12 shows visibility results for a different wave of $\lambda_{Y}=200 \mathrm{~km}$ and $\lambda_{Z}=25 \mathrm{~km}$, with $k_{Y}>0$ and $k_{Z}<0$ as before. Peak visibilities in Fig. 12a are larger $(\sim 40-45 \%)$ than those in Fig. 10a due to the longer vertical wavelength which is less smeared by the vertical weighting functions. Since the shorter cross-track wavelength is more prone to degraded detection by the broader off-nadir footprints, however, visibilities start decreasing with increasing $\left|\beta_{j}\right|$ earlier than in Fig. 10a. As in Fig. 10b, the cross-track visibility ratios $\hat{R}_{j, j_{*}}\left(k_{Y}, k_{Z}\right)$ in Fig. 12 b increase with increasing $\left|\beta_{j}\right|$ before rolling off towards more symmetric ratios at the outermost scan angles.

\section{Three-dimensional forward model simulations}

Here we simulate gravity wave detection more directly and in three dimensions by numerically evaluating Eq. (11) in the presence of a 3-D gravity wave temperature oscillation using the 3-D weighting functions from our AMSU-A radiance acquisition model. Our goal is to assess whether the two-dimensional horizontal coverage of the AMSU-A measurements in Fig. 6 can "image" the resolved gravity wave radiance perturbations in the horizontal.

We begin by specifying an idealized infinite threedimensional train of monochromatic gravity wave temperature oscillations of the form

$T^{\prime}(X, Y, Z)=T_{\text {peak }}(Z) \cos \left(k_{X} X+k_{Y} Y+k_{Z} Z\right)$,

where $T_{\text {peak }}$ is the peak wave temperature amplitude (potentially height dependent). We specify the wave's vertical wavelength $\lambda_{Z}=2 \pi /\left|k_{Z}\right|$, horizontal wavelength $\lambda_{h}=2 \pi / k_{h}$, and horizontal propagation azimuth $\varphi$ with respect to the AMSU-A viewing geometry axes $(\mathrm{X}, \mathrm{Y}, \mathrm{Z})$ in Fig. 1, such 
that $k_{X}= \pm 2 \pi / \lambda_{X}=k_{h} \cos \varphi$ and $k_{Y}= \pm 2 \pi / \lambda_{Y}=k_{h} \sin \varphi$. We then sample this temperature structure from orbit using the cyclical AMSU-A scanning pattern, acquiring brightness temperature perturbations $T_{B}^{\prime}\left(X_{j}, Y_{j}\right)$ by evaluating Eq. (11) using Eq. (15) as the 3-D temperature field. This calculation assumes that the background brightness temperature upon which these perturbations are superimposed (e.g., Fig. 7) can be perfectly extracted from the measurements: see Eckermann et al. (2006b) for examples of how this is done (imperfectly) in practice. Equation (11) is evaluated by rectangular integration after interpolating the model-generated weighting functions $W_{j}(X, Y, Z)$ onto a regular $(X, Y, Z)$ grid with $10 \mathrm{~km}$ horizontal and $0.5 \mathrm{~km}$ vertical grid spacings.

Figure 13 shows NOAA AMSU-A results for a wave of $\lambda_{h}=400 \mathrm{~km}$ and $\lambda_{Z}=12 \mathrm{~km}$, aligned at three different horizontal propagation azimuths $\varphi$. A constant peak amplitude $T_{\text {peak }}=5 \mathrm{~K}$ was used. Since there is no explicit dependence of these results on wave amplitude, instead of plotting absolute brightness temperatures $T_{B}^{\prime}\left(X_{j}, Y_{j}\right)$ we instead plot normalized visibility perturbations $T_{B}^{\prime}\left(X_{j}, Y_{j}\right) / T_{\text {peak }}$, to facilitate direct comparisons with the 2-D spectral visibilities in Sect. 4.

The 2-D weighting function spectra for this wave at $\varphi=90^{\circ}$ were profiled in Fig. 10a. Figure 13a shows results from our 3-D sampling of this wave for $\varphi=80^{\circ}$. As in Fig. 10b, there is weak but distinct cross-track asymmetry in the visibilities about the subsatellite point, with radiance visibility perturbation amplitudes of $\sim 13 \%$, a peak response at $Y \sim-500 \mathrm{~km}(j \sim 6)$, then a falloff to peak visibilities nearer $10 \%$ at the outermost scan angles. For our $5 \mathrm{~K}$ peak wave amplitude, visibility oscillations of $\pm 13 \%$ imply brightness temperature perturbations $T_{B}^{\prime}$ of $\pm 0.65 \mathrm{~K}$, well above our conservative nominal detectability threshold of $\pm 0.3 \mathrm{~K}$ discussed in Sect. 4.1. Similar amplitudes occur for the other two propagation directions $\varphi$ in Figs. 10b and c. The model results clearly indicate that NOAA AMSU-A Channel 9 radiances can image this particular gravity wave, regardless of its propagation angle with respect to the scanning geometry during the satellite overpass. The same holds for EOS Aqua, since its visibilities are greater at all beam positions than those of NOAA AMSU-A (see Fig. 10a).

However, the imaged wave structure in Fig. 13 changes noticeably with varying propagation direction $\varphi$. When the gravity wave horizontal wavenumber $\mathbf{K}_{\mathbf{h}}=\left(k_{X}, k_{Y}\right)$ is aligned roughly parallel to the cross-track $\mathrm{Y}$ axis $\left(\varphi=80^{\circ}\right.$ : Fig. 13a), horizontal wave phase lines (orthogonal to $\mathbf{K}_{\mathbf{h}}$ ) are well imaged by the near-nadir scans, but become poorly imaged at the outer scan angles due to the larger cross-track footprint diameters $\left(f_{Y}\right)_{j}$. This is entirely consistent with the falloff in 2-D spectral visibility for this wave $\left(\varphi=80^{\circ}\right)$ at large $\left|\beta_{j}\right|$ noted in Fig. 10a. For $\varphi=45^{\circ}$ (Fig. 13b) and $\varphi=350^{\circ}$ (Fig. 13c), the horizontal wave structure is imaged better at the outermost scan angles than for $\varphi=80^{\circ}$, since these wave orientations project longer wavelength components cross-track which are more easily resolved by the rel- atively wider cross-track footprint diameters $\left(f_{Y}\right)_{j}$. From Fig. 5, the along-track footprints $\left(f_{X}\right)_{j}$ at the far off-nadir beam positions are $\sim 56 \%$ the size of the cross-track footprints $\left(f_{Y}\right)_{j}$, and are oversampled in the along-track $(X)$ direction (see Fig. 6a). Both properties allow shorter horizontal wavelength structure to be imaged along-track compared to cross-track at the outermost scan angles, leading to better imaging of these latter wave orientations at the far off-nadir locations in Figs. 13b and $\mathrm{c}$ than in Fig. 13a.

The imaged wave fields yield phase and wavelength information. The imaged gravity wave phase lines in Figs. 13b and $\mathrm{c}$ show a noticeable curving or bowing, away from their intrinsic linear forms. This is produced by the upward displacement in the peaks of the vertical weighting functions with increasing scan angle in Figs. 3 and 4, due to the limb effect. This means that wave phase sampled at different beam positions $j$ varies not just due to the change in horizontal viewing location $\left(X_{j}, Y_{j}\right)$, but also with the vertical change in the altitude of the weighting function peaks. Since the former effect is dominant, the radiance perturbations do reproduce an approximate horizontal cross section of the wave oscillation pattern, such that the wavelengths in Fig. 13 reproduce the actual $400 \mathrm{~km}$ horizontal wavelength of this wave quite well. The curvature added to these phase lines is the smaller secondary influence of vertical phase changes induced by slightly different peak heights for the weighting functions $W_{j}(X, Y, Z)$ at each beam position $j$. Thus this curving of phase lines is the corresponding "limb effect" distortion for AMSU-A gravity wave measurements that "limb darkening" and "limb brightening" (Kidder and Vonder Haar, 1995) are for AMSU-A background temperature measurements (see Figs. $7 \mathrm{~b}$ and c).

Figure 14 shows results for the same three propagation azimuths as in Fig. 13, but for a gravity wave with a shorter horizontal wavelength $\left(\lambda_{h}=200 \mathrm{~km}\right)$ and longer vertical wavelength $\left(\lambda_{Z}=25 \mathrm{~km}\right)$. 2-D NOAA AMSU-A visibilities for this wave $\left(\varphi=90^{\circ}\right)$ in Fig. 12a showed peak visibility amplitudes of $\sim 43 \%$, decreasing rapidly with increasing $\left|\beta_{j}\right|$ at the outermost scan angles to values of $\sim 10-20 \%$. The $\varphi=80^{\circ}$ forward-modeled swath imagery for this wave in Fig. 14a reproduces these same trends in visibility.

Like the $\lambda_{h}=400 \mathrm{~km}$ wave in Fig. 13, the horizontal imaging of this wave's oscillations degrades with increasing $\left|\beta_{j}\right|$ more seriously for $\varphi=80^{\circ}$ (Fig. 14a) than for the other propagation azimuths in Figs. $14 \mathrm{~b}$ and c. When this $\lambda_{h}=200 \mathrm{~km}$ wave propagates cross-track (Fig. 14a), there is a highly nonuniform amplitude response cross-track in the imaged wave structure since only the narrow near-nadir AMSU-A footprints resolve it well. Conversely, when this wave propagates more nearly along track (Fig. 14c), the narrower footprints and oversampling along-track at the outermost scan angles yield a more uniform imaged response to this wave in the total swath coverage. As in Fig. 13, we also see curving of imaged wave phase lines for this wave, most noticeably at $\varphi=45^{\circ}$ in Fig. 14b. 

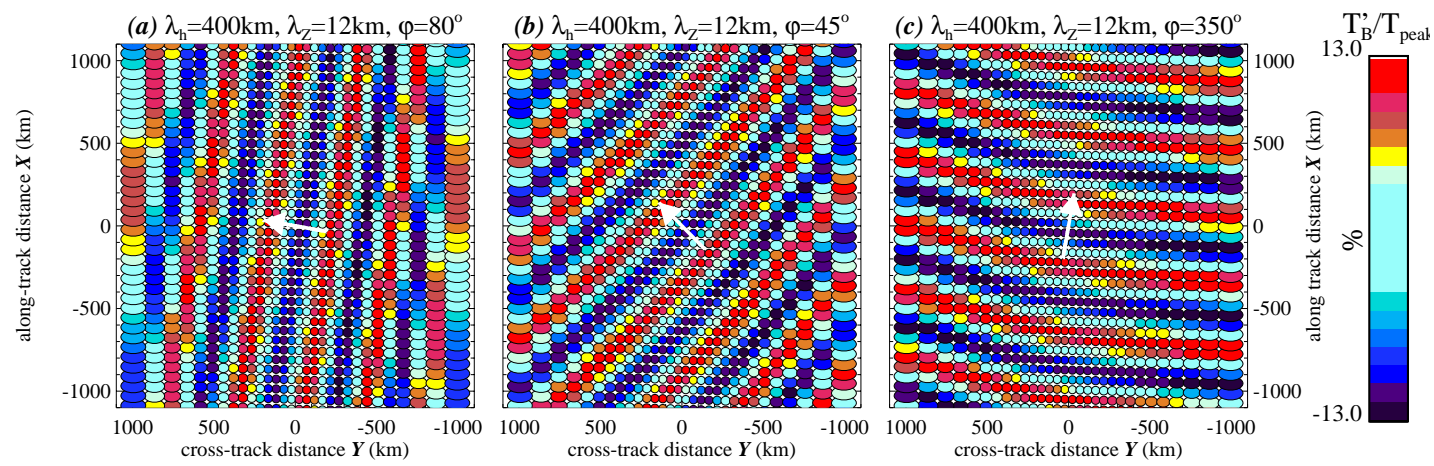

Fig. 13. (a) Relative brightness temperature perturbations (visibilities) $T_{B}^{\prime}\left(X_{j}, Y_{j}\right) / T_{\text {peak }}$ resulting from forward-modeled NOAA AMSU-A Channel 9 sampling of a gravity wave in Eq. (15) with $T_{\text {peak }}=5 \mathrm{~K}, \lambda_{h}=400 \mathrm{~km}, \lambda_{Z}=12 \mathrm{~km}$, and $\varphi$ values of (a) $80^{\circ}$, (b) $45^{\circ}$, and (c) $350^{\circ}$. The white vector at the center of each plot shows this direction of horizontal wave propagation $\varphi$. The color scale is the visibility amplitude expressed as a percentage, indicating a peak sensitivity of $\sim 13 \%$, or a maximum brightness temperature perturbation of $\sim 0.65 \mathrm{~K}$.
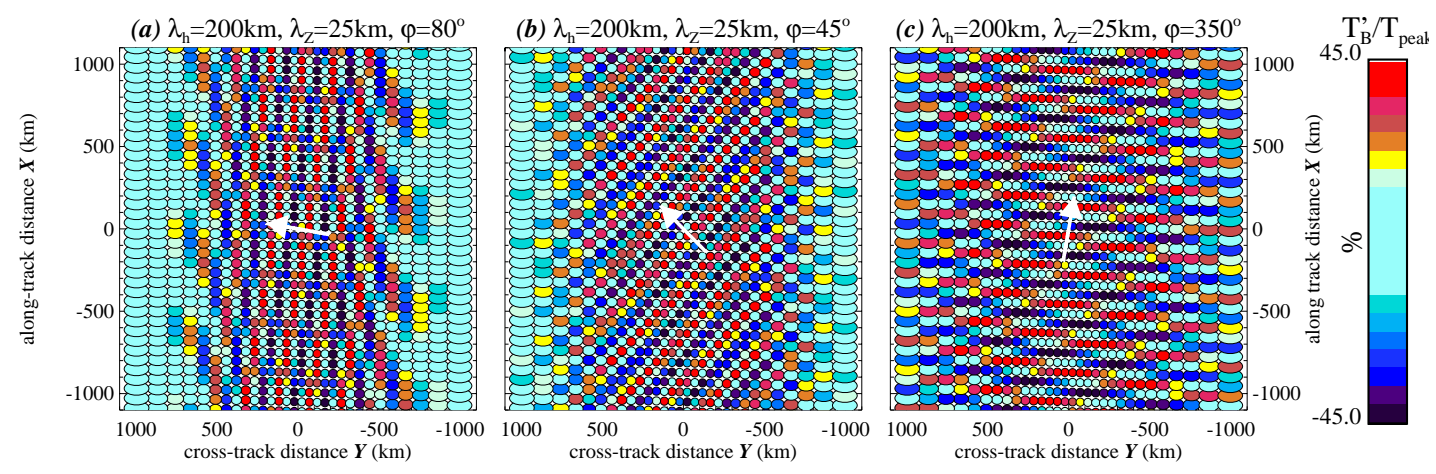

Fig. 14. Same presentation as in Fig. 13, but for a gravity wave with $\lambda_{h}=200 \mathrm{~km}, \lambda_{Z}=25 \mathrm{~km}$.

\section{Discussion}

To investigate the sensitivity of AMSU-A to atmospheric gravity waves, we have developed a simple numerical model of its in-orbit radiance acquisition. Of the six available stratospheric temperature channels, we focused on Channel 9. Since Channel 9 views a single $\mathrm{O}_{2}$ line and its 1$\mathrm{D}$ vertical weighting functions peak at $\sim 60-90 \mathrm{hPa}$, its radiative transfer should more closely approximate the purely pressure-broadened single Lorentz-line absorption model we developed in Sect. 3. Furthermore, Channel 9 has slightly narrower vertical weighting functions than the other stratospheric channels (Goldberg et al., 2001), making it (theoretically) more sensitive to gravity waves. Its lower altitude coverage also enables us to model any waves seen in these channel radiances with global and regional numerical prediction models, which can simulate both wave generation in the troposphere and the propagation of these waves into the lower stratosphere (e.g., Wu and Zhang, 2004; Eckermann et al., 2006b).

Using both spectral analysis of our model-derived 2-D weighting functions and numerical forward modeling using our 3-D weighting functions applied to 3-D gravity wave fields, we have shown that certain types of gravity wave oscillations appear in Channel 9 radiances as brightness temperature fluctuations with amplitudes well in excess of anticipated noise floors. While our results here are specific to this single channel, they argue for gravity wave detection in radiances from the other higher-altitude AMSU-A Channels 1014 as well, given the identical scanning pattern and similar footprint diameters and vertical weighting function widths (Goldberg et al., 2001). While noise floors (NE $\Delta \mathrm{T}$ ) increase somewhat for the higher-altitude channels (e.g., Lambrigtsen, 2003), so too do typical gravity wave temperature amplitudes and thus anticipated brightness temperature perturbations.

These findings support the observational results of $\mathrm{Wu}$ (2004), who analyzed radiance perturbations in AMSU-A Channel 13 radiances (which peak at $\sim 5 \mathrm{hPa}$ ) and found enhancements in radiance variances that had very similar geographical distributions to gravity wave-related enhancements in MLS radiances for the same observation period. In averaging AMSU-A radiances, $\mathrm{Wu}(2004)$ binned them within 6 different groups classified according to beam position. 


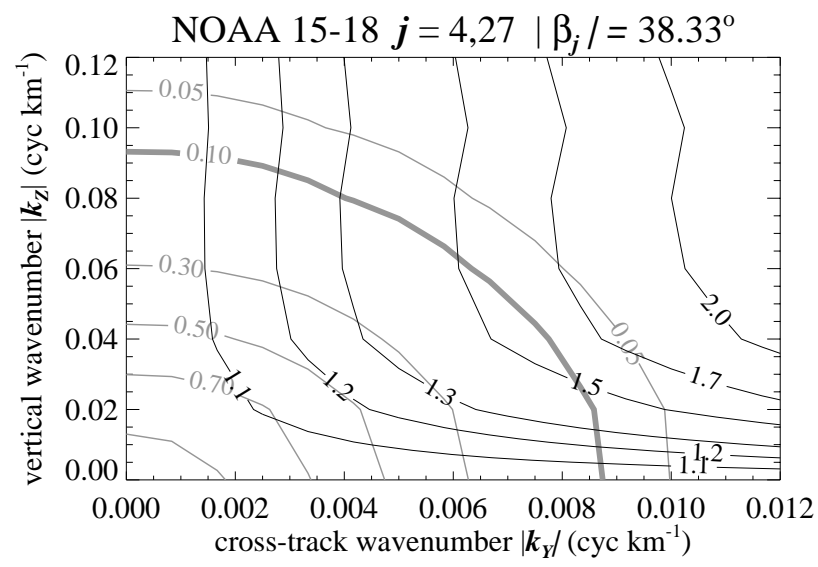

Fig. 15. Black contours show the spectral variance visibility ratio $\hat{R}_{j, j_{*}}^{2}\left(k_{Y}, k_{Z}\right)$ as a function of cross-track and vertical wavenumber for the NOAA AMSU-A $j=4, j_{*}=27$ conjugate beam pairing. Gray lines overlay absolute visibility contours of $\hat{W}_{j}\left(k_{Y}, k_{Z}\right)$ for the $j=4$ beam.

Groups 1 and 6 used data from the 10 farthest off-nadir beam positions ( $j=1-5$ and $j=26-30$ ), with group 1 incorporating data in which the beam pointed east $(\mathrm{Eg} 1)$, and group 6 using the remaining data in which the beam pointed west (Wg6). We denote these brightness temperature variances $\sigma_{\mathrm{Eg} 1}^{2}$ and $\sigma_{\mathrm{Wg} 6}^{2}$, respectively. On mapping these variances in the winter extratropical Southern Hemisphere, Wu (2004) found significant differences over the Andes and New Zealand, with $\sigma_{\mathrm{Eg} 1}^{2}$ typically a factor of 2 larger than $\sigma_{\mathrm{Wg} 6}^{2}$. This suggests crosstrack asymmetries in the response to the stratospheric gravity waves over these land masses.

In our analysis of modeled NOAA AMSU-A Channel 9 weighting functions, cross-track asymmetries in wave amplitude on the order of $10-15 \%$ (20-30\% in variance) were noted for two particular wave case studies. To study asymmetries over a wider range of wavelength pairings, Fig. 15 plots cross-track variance ratios $\hat{R}_{j, j_{*}}^{2}\left(k_{Y}, k_{Z}\right)$ for the NOAA AMSU-A $j=4, j_{*}=27$ conjugate beam pairing. Within regions of $>10 \%$ absolute visibility for $j=4$ (the thick solid gray contour in Fig. 15), we see cross-track variance ratios of up to 1.5, below the factor of 2 reported by Wu (2004) for Channel 13. Factors of 2 do occur in Fig. 15, but at larger wavenumbers where the visibilities are so small that we would anticipate little or no radiance signal above the noise floor unless those waves had very large amplitudes $T_{\text {peak. }}$. The larger anisotropies reported by $\mathrm{Wu}$ (2004) may result from his cross-track fitting method used to isolated wave fluctuations, which tends to isolate waves propagating crosstrack better than those propagating along track, since the latter project longer wavelengths cross-track (see Fig. 13c) that are harder to separate from mean cross-track limb effects (see Fig. 7c). In addition, differences in the weighting functions between Channels 13 and 9, or shortcomings in our simpli- fied treatment of radiative transfer and antenna specifics, may also yield underestimates in cross-track anisotropies.

Our 3-D forward model simulations in Sect. 5 also qualitatively confirm preliminary findings of Wu and Zhang (2004) that the two-dimensional horizontal coverage provided by AMSU-A's cross-track scanning pattern yields horizontal images of resolved gravity wave oscillations. A mesoscale model simulation by Wu and Zhang (2004) on the days and at the locations of their wave measurements produced gravity waves in the lower stratosphere with horizontal wavelengths of $\sim 300-500 \mathrm{~km}$ and vertical wavelengths of $\sim 7-15 \mathrm{~km}$ (see their Fig. 13). The approximate midpoint of this range corresponds to the $\lambda_{h}=400 \mathrm{~km}$ and $\lambda_{Z}=12 \mathrm{~km}$ wave we considered in both 2-D and 3-D model simulations (Figs. 10 and 13, respectively). Unfortunately, Wu and Zhang (2004) only plotted wave-induced divergence perturbations from their model runs, but they quote the largest temperature amplitudes in their model to be $\sim 5 \mathrm{~K}$. Our earlier modeling predicted peak visibilities of $\sim 13 \%$ for this wave and a maximum Channel 9 brightness temperature amplitude of $\sim 0.65 \mathrm{~K}$ for a $5 \mathrm{~K}$ wave temperature amplitude. This $0.65 \mathrm{~K}$ value is somewhat larger than the $\pm 0.3-0.5 \mathrm{~K}$ Channel 9 brightness temperature oscillations reported by $\mathrm{Wu}$ and Zhang (2004) (see their Figs. 5, 7 and 10). However, given the large uncertainties in the actual temperature amplitudes and vertical wavelengths for these wave oscillations and the strong senstivities of waveinduced Channel 9 radiance oscillations to uncertainties in both of these parameters (e.g., Fig. 8), these differences cannot be considered definitive. Definitive assessments require direct forward modeling of the full 3-D wave temperature fields into corresponding model-specified Channel 9 brightness temperatures, whose perturbation structures can then be compared directly to those observed. Such comparisons are the focus of the companion paper of Eckermann et al. (2006b). For a large-amplitude stratospheric gravity wave over southern Scandinavia, they report close agreement between observed Channel 9 radiance perturbations and those forward modeled using our 3-D weighting functions and 3-D temperature oscillations from numerical weather prediction models.

Our model results for AMSU-A also provide useful guidance for assessing the ability of future scanning microwave sensors to detect gravity waves. For example, the Advanced Technology Microwave Sounder (ATMS), slated to fly first on the National Polar-orbiting Operational Environmental Satellite System (NPOESS) Preparatory Project (NPP) and then on operational NPOESS platforms, will have similar scanning and temperature channel sensitivities to AMSU. Its smaller nominal nadir surface footprint diameters of $\sim 33 \mathrm{~km}$ should make ATMS more sensitive to shorter horizontal wavelengths. The Special Sensor Microwave Imager/Sounder (SSMIS) on the Defense Meteorological Satellite Program (DMSP) satellite launched in October 2003, which uses a conical scanning strategy (see Rosenkranz et al., 1997), yields radiances in its lower stratospheric 
temperature Channels 6 and 7 with footprint diameters of $\sim 37.5 \mathrm{~km}$ and nominal NE $\Delta \mathrm{T} \sim 0.4-0.5 \mathrm{~K}$. Our AMSU-A modeling indicates that the better horizontal resolution of SSMIS coupled with its narrower vertical weighting functions due to the fixed $45^{\circ}$ off-nadir beam position should allow this instrument to detect gravity wave fluctuations in its Channel 6-7 radiances, despite somewhat higher nominal noise floors compared to AMSU-A. Its fixed scan angle might also reduce the limb-effect distortion of phase lines noted here for AMSU-A.

\section{Summary and conclusions}

The modeling work outlined here has shown that gravity waves with long vertical wavelengths $\left(\lambda_{Z} \gtrsim 10 \mathrm{~km}\right)$, long horizontal wavelengths $\left(\lambda_{h} \gtrsim 150-200 \mathrm{~km}\right)$, and typical lower stratospheric temperature amplitudes $\left(T_{\text {peak }} \gtrsim 1-3 \mathrm{~K}\right)$ are resolved as perturbations to the lower stratospheric microwave radiances acquired by AMSU-A Channel 9 on both the NOAA meteorological satellites and NASA's EOS Aqua satellite. One entire AMSU-A scan cycle yields radiance measurements at 30 cross-track measurement locations spanning $\pm 800-1100 \mathrm{~km}$ either side of the satellite ground track. As the scan cycle repeats and the satellite motion sweeps out long two-dimensional "pushbroom" radiance images along track, the horizontal structure of these resolved waves is imaged in these radiance maps.

Our modeling has revealed some interesting instrumental effects that should aid interpretation of the gravity wave signals in these data. First, the amplitude of the gravity wave's radiance response varies systematically with crosstrack beam position $j$. As the cross-track viewing angle $\beta_{j}$ changes from near-nadir to further off-nadir, the AMSU-A weighting functions become broader cross-track and slightly narrower vertically due to the combined effects of antenna spreading, the limb effect and Earth curvature. These two width changes combine to yield 2-D weighting functions that are tilted farthest off-horizontal at the mid-range scan angles. These tilts lead to asymmetric responses to gravity waves when viewed at conjugate beam positions $j$ and $j_{*}$ $\left(\beta_{j_{*}}=-\beta_{j}\right)$, with amplitude differences typically on the order of $10-15 \%$. The narrower footprint diameters and greater sampling rates along track compared to cross track at the outermost scan angles allow gravity waves to be imaged more effectively when they propagate along track. The increase in altitude of the weighting function peak with increasing scan angle $\left|\beta_{j}\right|$ introduces a distorting curvature to the gravity wave phase lines imaged in these radiances, which can affect observational estimates of wave propagation directions or 3D wave structures. Nonetheless, for the waves we modeled, the radiance maps provide a fairly accurate (and valuable) direct measurement of the wave's horizontal wavelength.

In a companion paper, Eckermann et al. (2006b) apply the 3-D weighting function results and forward model methodol-

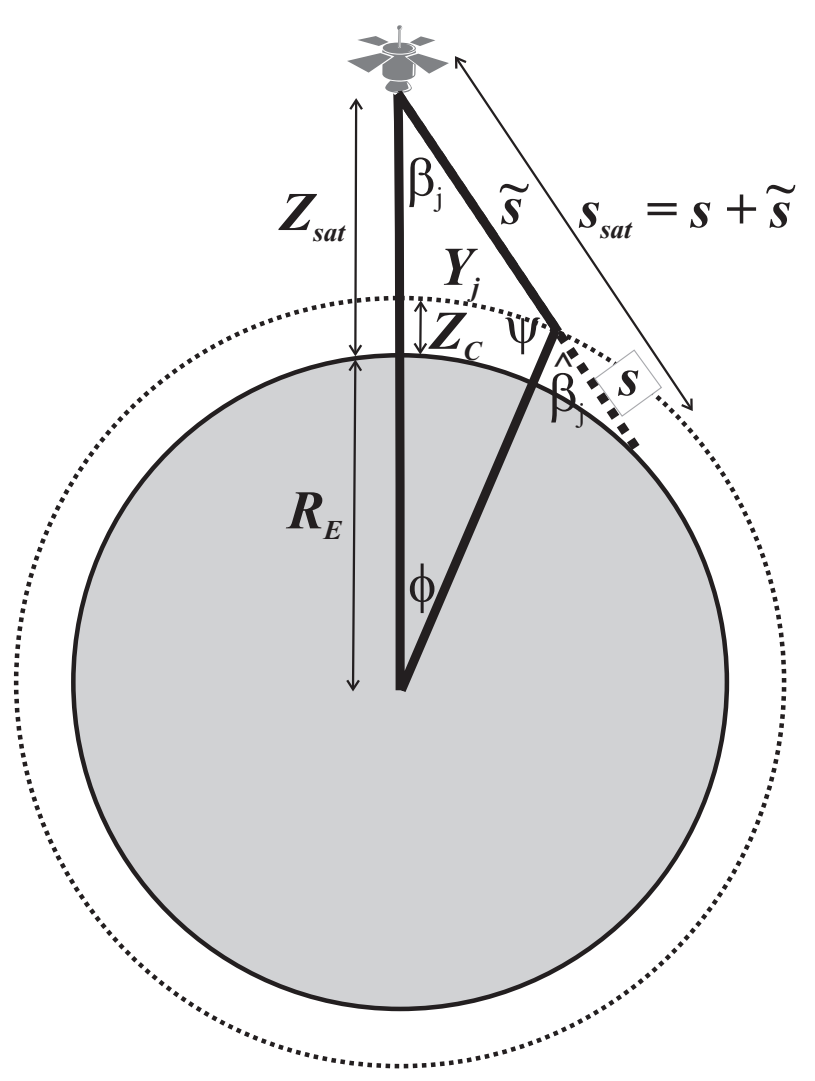

Fig. 16. AMSU-A observational geometry. The instrument views the atmosphere at a channel height $Z_{C}$ at a scan angle $\beta_{j}$. The lineof-sight distance from this measurement point to the satellite is $\tilde{s}$ and from the surface to this measurement point is $s . Y_{j}$ is the crosstrack swath distance from the subsatellite point to the measurement point. Other symbols are defined elsewhere in the text.

ogy we have developed here to an observational case study of a gravity wave over southern Scandinavia. This study combines AMSU-A Channel 9 radiance imagery and 3-D numerical model simulations of the wave field, in an attempt to fully characterize the wave and, via forward modeling, provides an observational validation of this paper's theoretical predictions of anticipated AMSU-A radiance responses to specific gravity wave-induced 3-D temperature structure.

\section{Appendix A}

\section{AMSU-A observational geometry and derivation of footprint diameter ratios}

The geometry is depicted in Fig. 16. From the law of sines,

$\hat{\beta}_{j}=\pi-\psi=\arcsin \left[\frac{\left(R_{E}+Z_{\mathrm{sat}}\right) \sin \beta_{j}}{R_{E}+Z_{C}}\right]$.

This is the off-nadir cross-track angle that the beam makes at a measurement point at altitude $Z_{C}$, differing from $\beta_{j}$ due 
to Earth curvature. Since $\phi+\beta_{j}+\psi=\pi$, then substituting Eq. (A1) yields Eq. (10), which in turn implies that

$\hat{\beta}_{j}=\beta_{j}+\phi\left(\beta_{j}\right)$.

The cross-track distance $Y_{j}$ from the subsatellite point to the measurement point at the altitude $Z_{C}$ is then just $\left(R_{E}+Z_{C}\right) \phi\left(\beta_{j}\right)$. Then we can estimate the cross-track halfpower footprint diameters to be

$$
\begin{aligned}
\left(f_{Y}\right)_{j}=\left[R_{E}+Z_{C}\right] & {\left[\phi\left(\beta_{j}+\frac{\beta_{\mathrm{HPBW}}}{2}\right)-\right.} \\
& \left.\phi\left(\beta_{j}-\frac{\beta_{\mathrm{HPBW}}}{2}\right)\right] .
\end{aligned}
$$

Along track, we estimate the footprint diameter using the line-of-sight ray distance $\tilde{s}$ between the satellite and measurement point and the width of the antenna polar diagram. The law of sines yields

$\tilde{s}=\frac{\left(R_{E}+Z_{C}\right) \sin \phi\left(\beta_{j}\right)}{\sin \beta_{j}}$,

whereupon, using a flat Earth approximation (adequate here given the small antenna width $\beta_{\mathrm{HPBW}}$ and no along-track tilting of the beam),

$\left(f_{X}\right)_{j}=2 \tilde{s} \tan \frac{\beta_{\mathrm{HPBW}}}{2}$.

Equations (A3)-(A5) yield Eq. (9), the along-track to cross-track footprint width ratio.

Acknowledgements. This research was supported by NASA's Earth Science and Geospace Sciences Programs, and by the Office of Naval Research.

Edited by: W. Ward

\section{References}

Alexander, M. J.: Interpretations of observed climatological patterns in stratospheric gravity wave variance, J. Geophys. Res., 103, 8627-8640, 1998.

Alexander, M. J. and Rosenlof, K. H.: Gravity-wave forcing in the stratosphere: Observational constraints from the Upper Atmosphere Research Satellite and implications for parameterization in global models, J. Geophys. Res., 108(D19), 4597, doi:10.1029/2003JD003373, 2003.

Dewan, E. M., Picard, R. H., O’Neil, R. R., Gardiner, H. A., Gibson, J., Mill, J. D., Richards, E., Kendra, M., and Gallery, W. O.: MSX satellite observations of thunderstorm-generated gravity waves in mid-wave infrared images of the upper stratosphere, Geophys. Res. Lett., 25, 939-942, 1998.

Eckermann, S. D. and Preusse, P.: Global measurements of stratospheric mountain waves from space, Science, 286, 1534-1537, 1999.
Eckermann, S. D., Hirota, I., and Hocking, W. K.: Gravity-wave and equatorial-wave morphology of the stratosphere derived from long-term rocket soundings, Quart. J. Roy. Meteorol. Soc., 121, 149-186, 1995.

Eckermann, S. D., Dörnbrack, A., Vosper, S. B., Flentje, H., Mahoney, M. J., Bui, T. P., and Carslaw, K. S.: Mountain waveinduced polar stratospheric cloud forecasts for aircraft science flights during SOLVE/THESEO 2000, Wea. Forecasting, 21, 4268, 2006a.

Eckermann, S. D., Wu, D. L., Doyle, J. D., Burris, J. F., McGee, T. J., Hostetler, C. A., Coy, L., Lawrence, B. N., Stephens, A., McCormack, J. P., and Hogan, T. F.: Imaging gravity waves in lower stratospheric AMSU-A radiances, Part: 2 Validation case study, Atmos. Chem. Phys., 6, 3343-3362, 2006 b.

Fetzer, E. J. and Gille, J. C.: Gravity wave variance in LIMS temperatures. Part I: Variability and comparison with background winds, J. Atmos. Sci., 51, 2461-2483, 1994.

Fritts, D. C. and Alexander, M. J.: Gravity wave dynamics and effects in the middle atmosphere, Rev. Geophys., 41(1), 1003, doi:10.1029/2001RG000106, 2003.

Goldberg, M. D., Crosby, D. S., and Zhou, L.: The limb adjustment of AMSU-A observations: methodology and validation, J. Appl. Meteorol., 40, 70-83, 2001.

Grody, N. C.: Remote sensing of the atmosphere from satellites using microwave radiometry, in: Atmospheric Remote Sensing by Microwave Radiometry, edited by: Janssen, M. A., Wiley, New York, 259-334, 1993.

Grody, N. C. and Pellegrino, P. P.: Synoptic-scale studies using the Nimbus 6 scanning microwave radiometer, J. Appl. Meteorol., 16, 816-826, 1977.

Hertzog, A., Vial, F., Dörnbrack, A., Eckermann, S. D., Knudsen, B. M., and Pommereau, J.-P.: In-situ observations of gravity waves and comparisons with numerical simulations during the SOLVE/THESEO 2000 campaign, J. Geophys. Res., 107(D20), 8292, doi:10.1029/2001JD001025, 2002.

Houghton, J. T., Taylor III, F. W., and Rodgers, C. D.: Remote sounding of atmospheres, Cambridge Univ. Press, 343pp, 1984.

Jiang, J. H., Eckermann, S. D., Wu, D. L., and Ma, J.: A search for mountain waves in MLS stratospheric limb radiances from the Northern Hemisphere: data analysis and global mountain wave modeling, J. Geophys. Res., 109, D03107, doi:10.1029/2003JD003974, 2004.

Kidder, S. Q. and Vonder Haar, T. H.: Satellite Meteorology: An Introduction, Academic Press, San Diego, 466pp, 1995.

Kidder, S. Q., Goldberg, M. D., Zehr, R. M., DeMaria, M., Purdom, J. F. W., Velden, C. S., Grody, N. C., and Kusselson, S. J.: Satellite analysis of tropical cyclones using the Advanced Microwave Sounding Unit (AMSU), Bull. Am. Meteorol. Soc., 81, 1241-1259, 2000.

Lambrigtsen, B. H.: Calibration of the AIRS microwave instruments, IEEE Trans. Geosci. Remote Sens., 41, 369-378, 2003.

Mann, G. W., Carslaw, K. S., Chipperfield, M. P., Davies, S., and Eckermann, S. D.: Large NAT particles and denitrification caused by mountain waves in the Arctic stratosphere, J. Geophys. Res., 110, D08202, doi:10.1029/2004JD005271, 2005.

McLandress, C., Alexander, M. J., and Wu, D.: Microwave limb sounder observations of gravity waves in the stratosphere: a climatology and interpretation, J. Geophys. Res., 105, 1947-1967, 2000 . 
Mo, T.: Prelaunch calibration of the Advanced Microwave Sounding Unit-A for NOAA-K, IEEE Trans. Microwave Theory Tech., 44, 1460-1469, 1996.

Mo, T.: AMSU-A antenna pattern corrections, IEEE Trans. Geosci. Remote Sens., 37, 103-112, 1999.

Parkinson, C. L.: Aqua: An Earth-observing satellite mission to examine water and other climate variables, IEEE Trans. Geosci. Remote Sens., 41, 173-183, 2003.

Poon, R. K. L.: A power law fit to oxygen absorption at $60 \mathrm{GHz}$ and its application to remote sensing of atmospheric temperature, Radio Sci., 15, 25-33, 1980.

Preusse, P., Eckermann, S. D., and Offermann, D.: Comparison of global distributions of zonal-mean gravity wave variance inferred from different satellite instruments, Geophys. Res. Lett., 27, 3877-3880, 2000.

Rosenkranz, P. W., Hutchison, K. D., Hardy, K. R., and Davis, M. S.: An assessment of the impact of satellite microwave sounder incidence angle and scan geometry on the accuracy of atmospheric temperature profile retrievals, J. Atmos. Ocean. Tech., 14, 488-494, 1997.

Staelin, D. H.: Inversion of passive microwave remote sensing data from satellites, in: Inversion Methods in Atmospheric Remote Sounding, edited by: Deepak, A., Academic, New York, 361394, 1977.

Tsuda, T., Nishida, M., Rocken, C., and Ware, R. H.: A global morphology of gravity wave activity in the stratosphere revealed by the GPS occultation data (GPS/MET), J. Geophys. Res., 105, 7257-7273, 2000.
Wang, L. and Geller, M. A.: Morphology of gravity-wave energy as observed from 4 years (1998-2001) of high vertical resolution U. S. radiosonde data, J. Geophys. Res., 108(D16), 4489, doi:10.1029/2002JD002786, 2003.

Waters, J. W., Kunzi, K. F., Pettyjohn, R. L., Poon, R. K. L., and Staelin, D. H.: Remote sensing of atmospheric temperature profiles with the Nimbus 5 microwave spectrometer, J. Atmos. Sci., 32, 1953-1969, 1975.

Wu, D. L.: Mesoscale gravity wave variances from AMSU-A radiances, Geophys. Res. Lett., 31, L112114, doi:10.1029/2004GL019562, 2004.

Wu, D. L. and Waters, J. W.: Satellite observations of atmospheric variances: A possible indication of gravity waves, Geophys. Res. Lett., 23, 3631-3634, 1996a.

Wu, D. L. and Waters, J. W.: Gravity-wave-scale temperature fluctuations seen by the UARS MLS, Geophys. Res. Lett., 23, 32893292, 1996b.

Wu, D. L. and Zhang, F.: A study of mesoscale gravity waves over the North Atlantic with satellite observations and a mesoscale model, J. Geophys. Res., 109, D22104, doi:10.1029/2004JD005090, 2004.

Wu, D. L., Preusse, P., Eckermann, S. D., Jiang, J. H., de la Torre Juarez, M., Coy, L., and Wang, D. Y.: Remote sounding of atmospheric gravity waves with satellite limb and nadir techniques, Adv. Space Res., 37, 2269-2277, 2006. 